\title{
The representative XMM-Newton cluster structure survey (REXCESS) of an X-ray luminosity selected galaxy cluster sample
}

\author{
H. Böhringer ${ }^{1}$, P. Schuecker ${ }^{1}$, G. W. Pratt ${ }^{1}$, M. Arnaud ${ }^{2}$, T. J. Ponman ${ }^{3}$, J. H. Croston ${ }^{4}$, S. Borgani ${ }^{5}$, R. G. Bower ${ }^{6}$, \\ U. G. Briel ${ }^{1}$, C. A. Collins ${ }^{7}$, M. Donahue ${ }^{8}$, W. R. Forman ${ }^{9}$, A. Finoguenov ${ }^{1}$, M. J. Geller ${ }^{9}$, L. Guzzo ${ }^{10}$, J. P. Henry ${ }^{11}$, \\ R. Kneissl ${ }^{12}$, J. J. Mohr ${ }^{13}$, K. Matsushita ${ }^{14}$, C. R. Mullis ${ }^{15}$, T. Ohashi ${ }^{16}$, K. Pedersen ${ }^{17}$, D. Pierini ${ }^{1}$, H. Quintana ${ }^{18}$, \\ S. Raychaudhury ${ }^{3}$, T. H. Reiprich ${ }^{19}$, A. K. Romer ${ }^{20}$, P. Rosati ${ }^{21}$, K. Sabirli ${ }^{22}$, R. F. Temple ${ }^{3}$, P. T. P. Viana ${ }^{23,24}$, \\ A. Vikhlinin ${ }^{9}$, G. M. Voit ${ }^{8}$, and Y.-Y. Zhang ${ }^{1}$ \\ (Affiliations can be found after the references)
}

Received 13 November 2006 / Accepted 5 March 2007

\begin{abstract}
Context. The largest uncertainty for cosmological studies using clusters of galaxies is introduced by our limited knowledge of the statistics of galaxy cluster structure, and of the scaling relations between observables and cluster mass.

Aims. To improve on this situation we have started an XMM-Newton Large Programme for the in-depth study of a representative sample of 33 galaxy clusters, selected in the redshift range $z=0.055$ to 0.183 from the REFLEX Cluster Survey, having X-ray luminosities above $0.4 \times 10^{44} h_{70}^{-2} \mathrm{erg} \mathrm{s}^{-1}$ in the $0.1-2.4 \mathrm{keV}$ band. This paper introduces the sample, compiles properties of the clusters, and provides detailed information on the sample selection function.

Methods. We describe the selection of a nearby galaxy cluster sample that makes optimal use of the XMM-Newton field-of-view, and provides nearly homogeneous X-ray luminosity coverage for the full range from poor clusters to the most massive objects in the Universe.

Results. For the clusters in the sample, X-ray fluxes are derived and compared to the previously obtained fluxes from the ROSAT All-Sky Survey. We find that the fluxes and the flux errors have been reliably determined in the ROSAT All-Sky Survey analysis used for the REFLEX Survey. We use the sample selection function documented in detail in this paper to determine the X-ray luminosity function, and compare it with the luminosity function of the entire REFLEX sample. We also discuss morphological peculiarities of some of the sample members.

Conclusions. The sample and some of the background data given in this introductory paper will be important for the application of these data in the detailed studies of cluster structure, to appear in forthcoming publications.
\end{abstract}

Key words. X-rays: galaxies: clusters - galaxies: clusters: general - cosmology: observations

\section{Introduction}

Galaxy clusters, as the largest well-defined dark matter haloes, are fundamental probes for the evolution of the cosmic largescale structure. Furthermore, they are ideal astrophysical laboratories for the study of numerous aspects of cosmic evolution, such as galaxy and star formation histories. The two major elements of such studies are (i) putting further constraints on cosmological models (e.g. Haiman et al. 2005, "white paper"), and (ii) the study of the evolution of the galaxies, both in the thermal and chemical imprint of the galaxies on the intracluster medium (e.g. Voit 2005; Borgani et al. 2005), and in the effect of the cluster environment on the formation and evolution of the galaxies (e.g. Croton et al. 2006).

With the recent progress in observational cosmology, a concordance cosmological model has become established, requiring two so far unknown ingredients - dark matter and dark energy (e.g. Perlmutter et al. 1999; Schmidt et al. 1999; Schuecker et al. 2003b; Spergel et al. 2006, 2003; Tegmark et al. 2006). Since the expansion of the Universe and the growth of structure depends very sensitively on both dark constituents, a detailed study of large-scale structure evolution in the recent past $(z \sim 2$ to 0 ), by means of galaxy clusters, can provide new insights into the nature of the dark components and provide tighter constraints on cosmological models. Galaxy clusters were among the first probes to be used to constrain dark energy models
(Wang \& Steinhardt 1998), and their importance as cosmological probes is increasingly being recognised (e.g. Rosati et al. 2002; Schuecker et al. 2003a,b; Majumdar \& Mohr 2004; Allen et al. 2004; Haiman et al. 2005).

Accurate mass estimates of the surveyed galaxy clusters are a prerequisite for such cosmological applications. X-ray observations are still the most attractive method to detect and characterize galaxy clusters. Not only is X-ray selection an approximate selection by cluster mass, due to the tight X-ray luminosity mass relation (Reiprich \& Böhringer 2002; Reiprich 2006), but it also provides a zeroth-order mass estimate through observables like the X-ray luminosity or X-ray temperature. For cosmological applications we need to have a precise knowledge of both the applied observable-mass relation and its intrinsic scatter. While such relations have been investigated for specially selected clusters (e.g. Ettori et al. 2004; Arnaud et al. 2005; Vikhlinin et al. 2006a; Zhang et al. 2006, 2007; Pedersen \& Dahle 2006), accurate calibrations for such scaling relations for a representative, unbiased sample of X-ray flux selected galaxy clusters at different epochs are still needed. Thus, a major goal of the present project is to provide a calibration baseline of representative scaling relations for the cluster population in the nearby Universe.

Quite apart cosmological applications, the form of the scaling relations between observables and mass, and the relations 
among different observables, provide important insights into the structure of galaxy clusters and the thermal structure of their intracluster medium. To first order, the scaling relations of observable cluster parameters as a function of cluster mass can be understood as self-similar, and have been successfully numerically simulated by purely gravitational structure growth (e.g. Bryan \& Norman 1998). However, second order effects, best observed in the low mass systems, show an altering of these simple self-similar relations as a consequence of energy input from other sources, related to galaxy and star formation processes and the cooling of dense intracluster gas regions (e.g. Kaiser 1991; Bower 1997; Ponman et al. 1999, 2003; Voit \& Bryan 2000; Voit et al. 2003; Pratt \& Arnaud 2005; Pratt et al. 2006). Therefore a detailed study of the scaling relations, in combination with the study of the enrichment of the intracluster medium by heavy elements, in conjunction with numerical modeling, provides important insights into the cosmic history of star formation and the processes that govern galaxy evolution (e.g. Pearce et al. 2000; Borgani et al. 2001, 2004; Muanwong et al. 2002; Kay et al. 2004; Finoguenov et al. 2003).

For both tests of cosmological models and studies of structure growth, a precise knowledge of the scaling relations, and well-measured cluster masses for large and systematically constructed cluster samples are the most important prerequisites. XMM-Newton, with its high sensitivity and the possibility of spatially resolved spectroscopy, provides the best means to approach this fundamental task. An inspection of the observational data on galaxy clusters in the XMM-Newton archive shows that it is impossible to construct a representative, statistically unbiased X-ray luminosity-selected sample, optimized for the XMM field-of-view, from the existing observations. Therefore we have successfully requested observing time for a comprehensive survey of X-ray structure of a representative cluster sample involving 33 galaxy clusters (where 3 data sets have been retrieved from the XMM-Newton archive).

A systematic study of clusters is absolutely necessary. There is for example a clear difference in the properties of clusters selected for their regularity, and those selected from flux or luminosity criteria, which include a wide range of morphologies. The scaling relations of X-ray luminosity and mass that apply to regular or compact cooling core clusters are expected to be different from those of dynamically less evolved clusters. This has been demonstrated for the X-ray luminosity - mass/temperature relation, showing that high central surface brightness objects, or objects described as classical cooling flows, have a significantly higher normalization than other clusters (O'Hara et al. 2006; Chen et al. 2006).

Thus, relations obtained for symmetric, apparently relaxed clusters will not be applicable to general cluster surveys. Therefore our primary goal is the calibration of the scaling relations for a statistical sample of clusters, selected by X-ray luminosity alone (the criterion most commonly used in cosmological applications of clusters). We also hope to establish the present data as a benchmark sample for studies in other wavelengths. In this context, contrary to most previous studies where researchers would choose the most regular clusters for an intercomparison, we want to provide a special incentive to also observe and reconstruct the more complex, apparently unrelaxed objects with different techniques of structure and mass measurements.

Therefore, some of the major goals of this project are to better characterize and understand (i) the relations of observables such as X-ray luminosity, temperature, and characteristic radius with cluster mass; (ii) the source of the scatter in these relations; (iii) the dynamical states of the clusters via inspection of temperature, entropy and pressure maps as well as by the comparison of X-ray and optical spectroscopic observations (guided by simulations); (iv) the statistics of cluster mergers and the frequency of cluster cooling cores as a function of cluster mass; both cosmologically very important diagnostics (e.g. Schuecker et al. 2001b); (v) entropy profiles of the clusters as probes of the thermal and star formation history in the clusters; (vi) metal abundances in clusters as a function of various observational parameters; and (vii) the variation of the cluster mass and mass-tolight ratio profiles.

We have also started a series of projects for observations of these clusters at other wavelengths, such as multicolourphotometry with the wide-field imaging camera, WFI, at the $2.2 \mathrm{~m} \mathrm{MPG/ESO}$ telescope, spectroscopic observations at Magellan, Sunyaev-Zeldovich observations with APEX, radio observations with GMRT (Giant Metrewave Radio Telescope), and high resolution X-ray observations with Chandra. A series of structure studies of these clusters is close to publication: analysis of the X-ray surface brightness and gas density distribution (Croston et al., for the novel analysis method see Croston et al. 2006), two-dimensional projected density, temperature, pseudopressure and pseudo-entropy maps (Finoguenov et al., similar to the analysis in Finoguenov et al. 2005, 2006), substructure analysis by a center shift method (Temple et al.), comparison of cluster structure of these observations with simulated clusters by means of a power ratio method (Böhringer et al.), and one study on the cluster temperature profiles, showing a high degree of universality of these profiles (outside the central regions) has been published (Pratt et al. 2007).

There is other work in progress to obtain similar information on representative cluster samples at other epochs, with which the present work will be combined in the future. An almost complete set of observations with XMM-Newton and Chandra has been performed on the brightest 63 galaxy clusters in the sky away from galactic plane, the HIFLUGCS sample (Reiprich \& Böhringer 2002), providing an account at even lower redshifts (although with a somewhat less ideal field-of-view criterion). The data reduction of this sample is in progress (Hudsen et al. and Reiprich et al. 2006). The REFLEX-DXL sample (Zhang et al. 2006) is a representative sample of the most X-ray luminous clusters in the redshift interval $z=0.27$ to 0.31 . After the launch of our project another XMM-Newton large programme was granted for the systematic study of cluster structure at intermediate redshifts of $z \sim 0.3-0.6$ (P.I. M. Arnaud). For even larger redshifts a systematic study of the RDCS clusters from Rosati et al. (1998, 2002, e.g. Ettori et al. 2004) and of the $160 \mathrm{deg}^{2}$ of Vikhlinin et al. (1998) is ongoing (e.g. Kotov \& Vikhlinin 2006). Together these studies shall provide a comprehensive view of the evolution of the cluster structure, and the corresponding scaling relations.

The derivation of the results presented in this paper is based on the "concordance cosmological model" with values of the normalized densities of $\Omega_{\mathrm{m}}=0.3, \Omega_{\Lambda}=0.7$ and a Hubble constant of $H_{0}=h_{70} 70 \mathrm{~km} \mathrm{~s}^{-1} \mathrm{Mpc}^{-1}$, if not explicitly stated otherwise.

\section{Sample construction}

\subsection{Primary considerations}

For the construction of an unbiased, X-ray selected cluster sample we use as a parent sample the REFLEX survey catalogue, which is presently the largest, well controlled cluster catalogue (Böhringer et al. 2004). The quality of the sample has been 


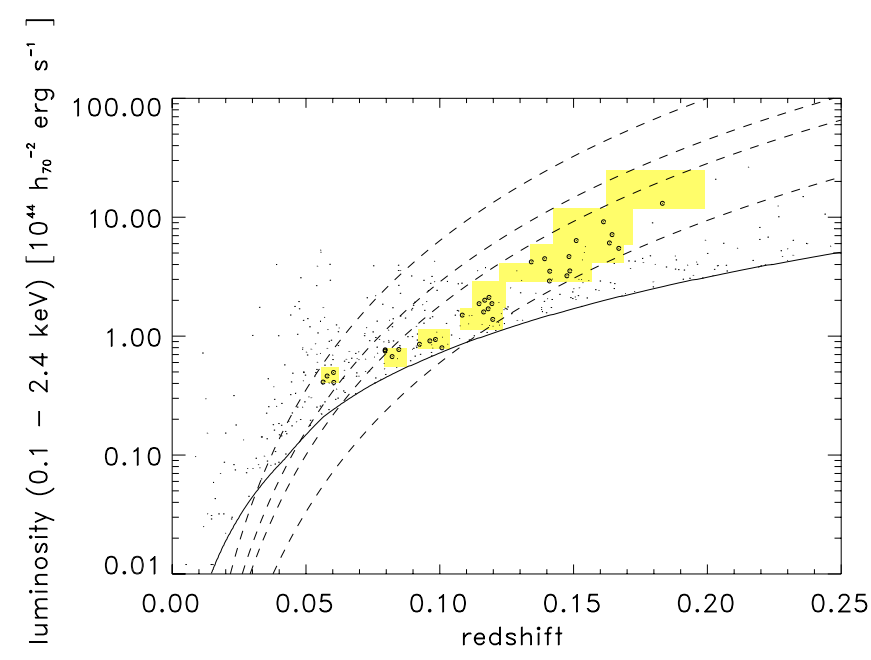

Fig. 1. X-ray luminosity-redshift distribution of the REFLEX sample (small dots: entire REFLEX sample including clusters with less than $30 \mathrm{cts}$ and $N_{\mathrm{H}}>6 \times 10^{20} \mathrm{~cm}^{-2}$ ), and the representative subsample (encircled dots) selected from the regions marked by colored boxes. The solid line indicates the survey flux limit. The dashed lines show the distances at which $r_{500}$ is $7,9,10$, and 12 arcmin (from right to left), for given X-ray luminosity, respectively.

demonstrated by showing that it can provide reliable measures of the large-scale structure without distorting artifacts (Collins et al. 2000; Schuecker et al. 2001a; Kerscher et al. 2003), yielding cosmological parameters in good agreement within the measurement uncertainties with the 3 year WMAP results (Schuecker et al. 2003a,b; Stanek et al. 2006; Spergel et al. 2006; note that this good agreement with the new WMAP data is also true for other cluster studies e.g. Voevodkin \& Vikhlinin 2004; Henry 2004). Moreover, the study of the galaxy cluster number density and the measured large-scale clustering provide consistent cosmological results.

REFLEX, based on the ROSAT All-Sky Survey (Trümper 1993), is a highly complete $(>90 \%)$ flux limited $\left(F_{\mathrm{X}}[0.1-2.4 \mathrm{keV}] \geq 3 \times 10^{-12} \mathrm{erg} \mathrm{s}^{-1} \mathrm{~cm}^{-2}\right)$ cluster sample, covering 4.24 ster in the southern extragalactic sky ( $\delta \leq 2.5 \mathrm{deg}$, $\left|b_{\mathrm{II}}\right| \geq 20 \mathrm{deg}$, with regions covered by the Magellanic clouds excluded - Böhringer et al. 2001). The variation of the sky coverage as a function of flux is small and is well documented in the REFLEX catalogue paper (Böhringer et al. 2004). There is a residual risk that a substantial part of the X-ray emission detected for these clusters comes from AGN in the cluster or in the background. We have estimated, however, that the fraction of clusters with severe contamination by AGN emission is smaller than $9 \%$.

The basic criteria for the selection of the present subsample are the following:

- We restrict the redshifts to $z \leq 0.2$ to obtain a census of the local Universe.

- The basic selection criterion is X-ray luminosity, with no preference for any particular morphological type. Thus the sample should be representative of any local, high quality, unbiased X-ray survey, a survey of the type applicable to cosmological model testing.

- To best assess the scaling relations, the selection has been designed to provide a close to homogenous coverage of the X-ray luminosity range. The chosen luminosity regime, $L_{\mathrm{X}}=0.407-20 \times 10^{44} h_{50}^{-2} \mathrm{erg} \mathrm{s}^{-1}$ in the $0.1-2.4 \mathrm{keV}$ rest frame band ${ }^{1}$, provides clusters with estimated temperatures above $2 \mathrm{keV}$. Thus the spectrum of selected objects covers the range from poor systems to the most massive clusters. Lower temperature systems, groups of galaxies, are excluded because their study requires a larger observational effort than the handful of additional data points that can be afforded here.

- We aim for a good global characterization of the clusters, and thus wish to detect cluster emission out to the fiducial radius $r_{500}$, the radius inside which the mean cluster mass density is 500 times the critical density of the Universe. This has been shown by simulations to provide one of the best measures of the size of the virialized dark matter system (Evrard et al. 1996).

- The distances of the objects are selected to optimally use the field-of-view, angular resolution, and photon collection power of the XMM-Newton observatory. For the data reduction we use the region of the target fields outside about 10-11 arcmin to assess the X-ray background of the observation. This is to enable a comparison of the properties of the target background and the background field to correct for background variations.

- We use well defined selection criteria such that the space density of the sample and any subset of it is well defined by the selection function.

These selection requirements cannot be met by a simple fluxlimit cut. In particular, to meet the condition of a nearly homogeneous luminosity coverage, we decided to draw the sample from the luminosity-redshift distribution in 8 luminosity bins containing a similar number of clusters. The FoV criterion then calls for a staircase like distribution of these bins in the $L_{X}$-redshift diagram shown in Fig. 1 (Each bin is almost volume limited with small corrections explained at the end of Sect. 2.2). To obtain sufficient statistics, the minimum number of clusters in such a sample is of the order of 30. The affordable amount of XMM-Newton observing time for deep enough studies of a cluster does not allow for a much larger number of targets. Therefore we decided to plan for the selection of four clusters per luminosity bin.

\subsection{Sample construction method}

We start the selection by choosing 9 luminosity bins of nearly equal logarithmic width, as defined in Table 1 and Fig. 1, with the calculation of the redshift for which the most luminous cluster in the bin has an apparent radius $r_{500}$ in the sky of 9 arcmin. This radius is calculated by means of the X-ray luminositytemperature relation taken from Ikebe et al. $(2002)^{2}$ :

$\frac{L_{\mathrm{X}}}{10^{44} \mathrm{erg} \mathrm{s}^{-1}}=0.02\left(\frac{T_{\mathrm{x}}}{1 \mathrm{keV}}\right)^{2.5} h_{100}^{-2}$.

We do not apply a redshift evolution correction here, since the HIFLUGCS sample studied by Ikebe et al. (2002) has a very similar redshift distribution as a function of luminosity to the present sample, and is therefore directly applicable. With the estimated temperature, $r_{500}$ can then be derived by

\footnotetext{
1 Originally selected as $L_{\mathrm{X}}=0.75-32 \times 10^{44} h_{50}^{-2} \mathrm{erg} \mathrm{s}^{-1}$ for an Einstein-De Sitter Universe.

2 All formulae are given for a concordance cosmology model as specified in Sect. 1, where $h_{100}=H_{0} / 100 \mathrm{~km} \mathrm{~s}^{-1} \mathrm{Mpc}^{-1}$.
} 
means of the temperature - radius relation from Arnaud et al. (2005):

$$
\begin{aligned}
r_{500} & =0.773 \mathrm{Mpc} h_{100}^{-1} E(z)^{-1}\left(\frac{T_{\mathrm{X}}}{5 \mathrm{keV}}\right)^{0.57} \\
& =0.753 \mathrm{Mpc} h_{100}^{-0.544} E(z)^{-1}\left(\frac{L_{\mathrm{X}}}{10^{44} \mathrm{erg} \mathrm{s}^{-1}}\right)^{0.228}
\end{aligned}
$$

with $E(z)=h(z) / h_{0}$. The second-lowest dashed line in Fig. 1 corresponds to the relation of X-ray luminosity and redshift for which $r_{500}$ appears as 9 arcmin. Then we select the bins as follows:

- (1) The upper left corner of bins 5-7 are defined by the 9 arcmin radius line, effectively fixing the lower redshift boundary. We collect the 4 clusters we wish to have in the bin by increasing the redshift. The outer redshift boundary of the bin is defined by the midpoint between the last cluster in the sample and the first cluster outside.

- (2) For practical reasons we have not strictly applied this rule to all bins. Using this criterion, luminosity bins $2-4$ extend very close to the nominal flux limit (the solid curve in Fig. 1). For these bins we decided to start filling the bins from the high redshift side, touching the flux limit with the lower right corner of the bin, and filling the bin by collecting clusters at lower redshifts. The inner boundary of the redshift bin is defined by the midpoint in redshift between the last cluster in the bin and the first cluster at lower redshift outside.

- (3) The lowest luminosity objects (bin 1) have a lower surface brightness, and we do not expect very much emission at large radii. To better use the field-of-view of XMM-Newton, and to increase the flux from these clusters, we moved this lowest luminosity bin to the limit where the most luminous cluster would have an apparent $r_{500}$ of 12 arcmin.

- (4) For the most luminous clusters (bins 8 and 9), which are very rare, we increased the search volume at low redshift by allowing the most luminous cluster to have an $r_{500}$ of 10 arcmin. We also relax the interstellar column density constraints and allow values of $N_{\mathrm{H}}$ larger than $6 \times 10^{20} \mathrm{~cm}^{-2}$.

- (5) In bin 9, we find only one cluster in the region between an $r_{500}$ of 10 arcmin and a redshift of 0.2. This cluster is A1689, which has already been observed with XMM-Newton and the data are available in the archive. The outer redshift boundary of this bin is again determined by the midpoint to the next object at higher redshift.

The original sample was constructed from a preliminary REFLEX catalogue. Between the first complete catalogue construction and the final revision and subsequent publication of the catalogue in Böhringer et al. (2004), a series of new galaxy redshifts became available in the literature, publicly available data bases, and through our own observations. This led to improved cluster redshifts. Since the redshift boundaries of our relatively small cluster sample are very tight, there was an unavoidable scatter of objects across the boundaries. We checked the typical changes in the redshifts of the REFLEX sample that cause the scattering of the clusters in redshift space, and found that about $10-15 \%$ of the clusters experienced shifts of $400-2000 \mathrm{~km} \mathrm{~s}^{-1}$, resulting in the above described effect.

This has led us to reconstruct the selection scheme by applying the same criteria such that the originally-selected clusters are still contained in the survey volume. The mid point rule for the outer or inner redshift boundary gives us the flexibility to reconstruct using the new redshifts. The price paid is that a small number of new clusters appear in the bins, which then have to be corrected for in the selection function. With this revision we also changed the luminosity values from a critical density universe to a concordance cosmological system $\left(\Omega_{\mathrm{m}}=0.3, \Omega_{\Lambda}=0.7\right.$, and $h_{70}=1$ ), which results in the inclusion of two additional clusters inside the bins. The advantage of this reconstruction is that the new survey selection function is fully compatible and reproducible with the published REFLEX data set. Figure 1 provides an account for the complete selection scheme. The resultant redshift boundaries are listed in Table 1 . The total number of clusters ending up in the bins is given in Col. 7 of this table, and the extra clusters are explicitly listed in Table 4.

Two further selection criteria are important: (i) to avoid importing galaxy clusters with lower quality detection parameters (flux error, extent parameter, etc.) we have only included galaxy clusters which contained more than 30 detected counts in the ROSAT All-Sky Survey. The same cut was made in the construction of the X-ray luminosity function in Böhringer et al. (2002). In addition, (ii) to obtain good X-ray spectra with a wide spectral coverage, we only selected clusters in sky areas where the hydrogen column density, $N_{\mathrm{H}}$, measured at $21 \mathrm{~cm}$ (Dickey \& Lockman 1990), is smaller than $6 \times 10^{20} \mathrm{~cm}^{-2}$. This criterion was not applied to the most luminous clusters in bin number 8 , since there are only few such objects. In addition the spectra of these clusters have high expected X-ray temperatures, and will be less influenced by the hydrogen column density than those with lower temperatures.

To determine the selection volume associated with each cluster we apply the following steps. To take into account of the $N_{\mathrm{H}}$ selection, we have inspected the fraction of the sky region in the REFLEX area with $N_{\mathrm{H}} \geq 6 \times 10^{20} \mathrm{~cm}^{-2}$. This fraction is slightly dependent on the flux limit of the sky region, such that less sensitive regions have on average higher column densities. The fraction of sky area above our $N_{\mathrm{H}}$-cut is about 22-23\% over more than $90 \%$ of the sky. Only in a smaller less sensitive area is it slightly larger. Thus we correct the sky coverage by a factor of 0.775 for all bins except for bin 8 and 9, as shown in Col. 9 of Table 1.

The other condition, that we should have a detection of at least 30 photons, further reduces the sky coverage, since in only $78 \%$ of the REFLEX Survey area is the nominal flux limit reached for the detection of 30 photons (as explained in detail in the REFLEX sample construction paper, Böhringer et al. 2001). This effect is especially important for those bins which are close to the nominal flux limit. Therefore we have to determine the mean "volume coverage" of each bin as a function of the luminosity and redshift range within the $\operatorname{bin}^{3}$. This average volume coverage per bin is different for each bin and the correction factors are given in Table 1. Both corrections are small but significant, so that it is important to include them. The empirical nature of these corrections will introduce only minor second order uncertainties, which are definitely only of the order of one percent.

The final step is the normalization of the selection function. Most bins contain only the four clusters we initially selected. In this case we determine the cluster density for this luminosity bin by the inverse volume, multiplied by four (no multiplication

3 Each bin constitutes an almost volume limited subsample. The ROSAT Survey contains, however, a few regions with reduced sensitivity, where the survey becomes flux-limited. Therefore each grid point of luminosity and redshift within the bin has a "sky coverage" of slightly less than $100 \%$. The fractional sky coverage averaged over all $L_{\mathrm{x}}$, $z$ grid points of the bin yields the mean "volume coverage". 
Table 1. The luminosity-redshift bins used for the selection of the sample (for $H_{0}=70 \mathrm{~km} \mathrm{~s}^{-1} \mathrm{Mpc}^{-1}, \Omega_{\mathrm{m}}=0.3, \Omega_{\Lambda}=0.7$ ).

\begin{tabular}{llllllllllll}
\hline \hline Bin no. & $N_{\mathrm{Cl}}$ & $L_{\mathrm{X}}(\min )$ & $L_{\mathrm{X}}(\max )$ & $z_{\min }$ & $z_{\max }$ & $\begin{array}{l}N_{\text {tot }} \\
\text { vol.cov. } \\
\text { correction }^{a}\end{array}$ & $\begin{array}{l}\text { skycov. } \\
\text { correction }\end{array}$ \\
$(1)$ & $(2)$ & $(3)$ & $(4)$ & $(5)$ & $(6)$ & $(7)$ & $(8)$ & $(9)$ & $(10)$ & $(11)$ \\
\hline 1 & 4 & 0.407 & 0.55 & 0.0555 & 0.06215 & 4 & 0.94 & 0.775 & $5.28(-6)$ & $4.95(-6)$ \\
2 & 4 & 0.55 & 0.78 & 0.0794 & 0.0877 & 4 & 0.86 & 0.775 & $3.33(-6)$ & $3.12(-6)$ \\
3 & 4 & 0.78 & 1.13 & 0.0920 & 0.1037 & 7 & 0.87 & 0.775 & $8.72(-7)$ & $7.39(-7)$ \\
4 & 4 & 1.13 & 1.71 & 0.1077 & 0.12105 & 8 & 0.88 & 0.775 & $3.17(-7)$ & $2.80(-7)$ \\
5 & 4 & 1.71 & 2.88 & 0.1122 & 0.1248 & 4 & 0.94 & 0.775 & $8.87(-8)$ & $8.39(-8)$ \\
6 & 4 & 2.88 & 4.10 & 0.1224 & 0.15215 & 4 & 0.95 & 0.775 & $2.73(-8)$ & $2.53(-8)$ \\
7 & 4 & 4.10 & 5.90 & 0.1337 & 0.16875 & 5 & 0.97 & 0.775 & $1.60(-8)$ & $1.20(-8)$ \\
8 & 4 & 5.90 & 11.9 & 0.1423 & 0.1719 & 4 & 1.00 & 1.0 & $3.17(-9)$ & $1.33(-9)$ \\
9 & 1 & 11.9 & 20 & 0.1623 & 0.19925 & 1 & 1.00 & 1.0 & $3.66(-10)$ & $1.62(-10)$ \\
\hline
\end{tabular}

$N_{\mathrm{Cl}}$ is the number of sample clusters per bin.

$L_{\mathrm{X}}(\min ), L_{\mathrm{X}}(\max ), z_{\min }$, and $z_{\max }$ give the luminosity (in $10^{44} \mathrm{erg} \mathrm{s}^{-1}[0.1-2.4 \mathrm{keV}]$ ) and redshift boundaries of the bins, respectively.

$N_{\text {tot }}$ is the total number of clusters per bin including the unobserved ones.

${ }^{a}$ gives the mean volume coverage fraction of the REFLEX survey above the flux limit, for the detection of 30 photons for the bins.

${ }^{b}$ fraction of sky coverage for an interstellar column density $N_{\mathrm{H}} \leq 6 \times 10^{20} \mathrm{~cm}^{-2}$.

${ }^{c}$ cluster density $\left(\mathrm{Mpc}^{-3}\right)$ determined from the selection function derived in Sect. 2.2, where the number in brackets gives the exponent of 10.

${ }^{d}$ cluster density $\left(\mathrm{Mpc}^{-3}\right)$ from the alternative method used to determine the selection function.

Table 2. The luminosity-redshift bins used for the alternatively constructed test selection function.

\begin{tabular}{|c|c|c|c|c|c|c|c|c|}
\hline $\begin{array}{l}\text { Bin no. } \\
\text { (1) }\end{array}$ & $\begin{array}{l}N_{\mathrm{Cl}} \\
(2)\end{array}$ & $\begin{array}{l}L_{X}(\min ) \\
(3)\end{array}$ & $\begin{array}{l}L_{X}(\max ) \\
(4)\end{array}$ & $\begin{array}{l}z_{\min } \\
(5)\end{array}$ & $\begin{array}{l}z_{\max } \\
(6)\end{array}$ & $\begin{array}{l}z(\text { excl.) } \\
(7)\end{array}$ & $\begin{array}{l}z(\text { excl.) } \\
(8)\end{array}$ & $\begin{array}{l}z(\text { excl.) } \\
(9)\end{array}$ \\
\hline 1 & 4 & 0.75 & 1.0 & 0.0550 & 0.06215 & & \multirow{9}{*}{$\begin{array}{l}(0.09665-0.09795) \\
(0.11835-0.1195)\end{array}$} & \multirow{9}{*}{$(0.09865-0.09985)$} \\
\hline 2 & 4 & 1.0 & 1.4 & 0.0794 & 0.08725 & & & \\
\hline 3 & 4 & 1.4 & 2.0 & 0.0920 & 0.1041 & $(0.0926-0.09455)$ & & \\
\hline 4 & 4 & 2.0 & 3.0 & 0.1077 & 0.12105 & $(0.1102-0.1131)$ & & \\
\hline 5 & 4 & 3.0 & 5.0 & 0.11145 & 0.1248 & & & \\
\hline 6 & 4 & 5.0 & 7.0 & 0.1195 & 0.15215 & $(0.1195-0.15685)^{*}$ & & \\
\hline 7 & 4 & 7.0 & 10 & 0.1161 & 0.16875 & $(0.15135-0.16065)$ & & \\
\hline 8 & 4 & 10 & 20 & 0.0 & 0.1719 & & & \\
\hline 9 & 1 & 20 & 32 & 0.0 & 0.19925 & & & \\
\hline
\end{tabular}

The first 6 columns have the same meaning as those in Table 2, where the luminosity bins are now given for a critical density

Universe with a Hubble constant of $h_{100}=0.5$.

$z_{\text {excl }}$ give the redshift intervals of the regions to be excluded due to unobserved clusters scattered into the bins.

* gives the total redshift interval of the bins for a Universe with critical density.

in the case of bin 9). In bins 3, 4, and 7, where we find new, unobserved clusters in the reconstructed sample due to redshift scattering, we normalize by the total number of clusters in the bin.

The information provided for the selection function makes it possible to determine the distribution function of any property of the clusters in the sample. The estimated density of clusters for a given luminosity interval is the inverse of the selection volume multiplied by the number of clusters found in the luminosity bin. This calculation can also be restricted to a specific type of cluster. In this case for the density calculation the inverse volume is simply multiplied by the number of clusters of this type in the luminosity bin. We will demonstrate how this is done for the luminosity function as an example in Sect. 7. For further work with the REXCESS sample, the construction of the temperature function, the mass function, but also more peculiar functions like the cool core gas mass function, this procedure will be important. This range of applications is precisely the strength of the XMM-Newton Legacy Program. The dataset provides, for the first time, a representative X-ray cluster sample, observed deep enough to provide a wealth of parameters on cluster structure (allowing for a complete cluster coverage by the XMM-Newton field-of-view), and at the same time large enough to allow the construction of meaningful statistics.

\subsection{Alternative experimental sample selection}

The proper statistical modeling of such a survey selection function is only to first order approximation a trivial task. In the presence of substantial measurement errors, or correlation uncertainties for a cluster property distribution function other than the luminosity function, scattering effects have to be accounted for. These effects correspond to the so-called Malmquist bias in flux limited samples. In the present case these effects are more complicated, and are best treated by Monte Carlo simulations. For a proper accounting one may not only consider the boundary migration effects, but also the boundary selection itself, since e.g. the midpoint rule selection depends on the statistics of the cluster distribution in luminosity and redshift space. This should also be included in the Monte Carlo simulations.

Details of such an analysis will be considered in a future paper concerning the temperature or mass function construction. Here we adopt a didactical point of view, and attempt to illustrate the variance in the results due to the statistics of the boundary 
Table 3. The REFLEX XMM Large Program cluster sample.

\begin{tabular}{|c|c|c|c|c|c|c|c|c|c|c|c|c|}
\hline $\begin{array}{l}\text { Name } \\
(1)\end{array}$ & $\begin{array}{l}\text { Alt.name } \\
\text { (2) }\end{array}$ & $\begin{array}{r}\mathrm{RA}(2000) \\
(3)\end{array}$ & $\begin{array}{r}\operatorname{Dec}(2000) \\
(4)\end{array}$ & $\begin{array}{r}z \\
(5)\end{array}$ & $\begin{array}{l}N_{\text {gal }} . \\
\text { (6) }\end{array}$ & $\begin{array}{l}F_{\mathrm{x}} \\
(7)\end{array}$ & $\begin{array}{r}\text { Error } \\
(8)\end{array}$ & $\begin{array}{l}L_{\mathrm{x}} \\
(9)\end{array}$ & $\begin{array}{r}R_{\mathrm{ap}} \\
(10)\end{array}$ & $\begin{array}{r}L_{\mathrm{x}}^{*} \\
(11)\end{array}$ & $\begin{array}{r}N_{\mathrm{H}} \\
(12)\end{array}$ & $\begin{array}{r}L_{\mathrm{x}}-\text { bin } \\
(13)\end{array}$ \\
\hline RX CJ0003.8+0203 & A2700 & 000350.6 & +020348 & 0.0924 & 9 & 4.155 & 18.8 & 0.855 & 8.5 & 0.929 & 3.0 & 3 \\
\hline RX CJ0006.0-3443 & A2721 & 000603.0 & -344327 & 0.1147 & 75 & 5.832 & 13.6 & 1.875 & 10.0 & 1.995 & 1.2 & 5 \\
\hline RX CJ0020.7-2542 & A0022 & 002042.8 & -254237 & 0.1410 & 3 & 5.910 & 12.1 & 2.909 & 7.5 & 3.232 & 2.3 & 6 \\
\hline RX CJ0049.4-2931 & S0084 & 004924.0 & -293128 & 0.1084 & 18 & 5.228 & 16.0 & 1.503 & 11.0 & 1.566 & 1.8 & 4 \\
\hline RX CJ0145.0 & A2941 & 02.3 & -530050 & 0.1168 & 4 & 6.028 & 16.0 & 2.005 & 7.5 & 2.253 & 2.3 & 5 \\
\hline $\mathrm{RXC}$ & A2984 & 25.5 & 712 & 0.1008 & 6 & 3.222 & 11.5 & 0.798 & 8.0 & 0.858 & 1.4 & 3 \\
\hline RX CJ0225.1 & & 022510.5 & -292826 & 0.0604 & 17 & 4.736 & 23.4 & 0.408 & 12.0 & 0.434 & 1.7 & 1 \\
\hline 4112 & S0384 & 45.7 & -411227 & 0.0603 & 1 & 5.763 & 18.9 & 0.495 & 11.5 & 0.532 & 1.9 & 1 \\
\hline RX CJ0547.6 & A3364 & 38.2 & -315231 & 0.1483 & 10 & 8.526 & 7.5 & 4.667 & 17.5 & 4.667 & 2.0 & 7 \\
\hline RX CJ0605.8-3518 & A3378 & 060552.8 & -351802 & 0.1392 & 2 & 9.393 & 6.2 & 4.478 & 12.5 & 4.665 & 4.3 & 7 \\
\hline RX CJ0616.8-4748 & & 061653.6 & -474818 & 0.1164 & 1 & 4.813 & 9.8 & 1.597 & 14.0 & 1.613 & 4.8 & 4 \\
\hline RX CJ0645.4-5413 & A3404 & 064529.3 & -541308 & 0.1644 & 2 & 10.597 & 7.9 & 7.139 & 13.0 & 7.360 & 6.6 & 8 \\
\hline RX CJ0821.8+0112 & A0653 & 082151.7 & +011242 & 0.0822 & 6 & 4.142 & 19.2 & 0.673 & 12.0 & 0.701 & 4.2 & 2 \\
\hline RX CJ0956.4-1004 & A0901/2 & 095626.4 & -100412 & 0.1634 & 9 & 9.115 & 9.6 & 6.077 & 17.0 & 6.077 & 5.1 & 8 \\
\hline-1103 & A0907 & 095822.1 & -110335 & 0.1669 & 2 & 7.833 & 8.3 & 5.472 & 8.5 & 5.948 & 5.1 & 7 \\
\hline RX CJ1044.5-0704 & A1084 & 104433.0 & -070422 & 0.1342 & 6 & 9.451 & 12.2 & 4.213 & 7.0 & 4.899 & 3.4 & 7 \\
\hline RX CJ1141.4-1216 & A1348 & 114124.3 & -121620 & 0.1195 & 6 & 5.344 & 12.3 & 1.877 & 7.0 & 2.109 & 3.3 & 5 \\
\hline RX CJ1236.7-3354 & S0700 & 123644.7 & -335410 & 0.0796 & 4 & 4.932 & 19.8 & 0.749 & 8.5 & 0.832 & 5.6 & 2 \\
\hline RX CJ1302.8-0230 & A1663 & 50.7 & -023022 & 0.0847 & 3 & 4.460 & 21.9 & 0.772 & 12.0 & 0.804 & 1.7 & 2 \\
\hline 0120 & A1689 & 30.0 & 007 & 0.1832 & 66 & 15.332 & 8.0 & 13.088 & 10.5 & 14.073 & 1.8 & 9 \\
\hline & AGN & 43.9 & -334317 & 0.1142 & 1 & 4.021 & 17.3 & 1.280 & 7.0 & 1.422 & 4.8 & 4 \\
\hline RX CJ1516.3+0005 & A2050 & 1619.2 & +000552 & 0.1181 & 17 & 4.956 & 13.8 & 1.697 & 7.5 & 1.886 & 4.6 & 4 \\
\hline RX CJ1516.5-0056 & A2051 & 1634.0 & -005656 & 0.1198 & 7 & 3.918 & 61.7 & 1.383 & 13.0 & 1.397 & 5.5 & 4 \\
\hline RX CJ2014.8 & & 201449.7 & -243030 & 0.1612 & 2 & 14.040 & 13.4 & 9.157 & 6.5 & 11.033 & 7.4 & 8 \\
\hline X CJ2023.0-2056 & S0868 & 202301.6 & -205655 & 0.0564 & 2 & 5.497 & 17.8 & 0.411 & 8.5 & 0.467 & 5.6 & 1 \\
\hline RX CJ2048.1-1750 & A2328 & 204810.6 & -175038 & 0.1475 & 3 & 5.930 & 15.2 & 3.215 & 10.5 & 3.349 & 4.8 & 6 \\
\hline RX CJ2129.8-5048 & A3771 & 212951.0 & -504804 & 0.0796 & 2 & 5.051 & 66.2 & 0.767 & 11.5 & 0.807 & 2.2 & 2 \\
\hline RX CJ2149.1-3041 & A3814 & 214907.4 & -304155 & 0.1184 & 19 & 6.182 & 12.9 & 2.117 & 9.5 & 2.276 & 2.3 & 5 \\
\hline RX CJ2152.2-1942 & A2384(B) & 215214.2 & -194220 & 0.0963 & 4 & 4.059 & 30.0 & 0.912 & 6.0 & 1.060 & 3.0 & 3 \\
\hline RX CJ2157.4-0747 & A2399 & 215725.8 & -074741 & 0.0579 & 8 & 5.851 & 19.0 & 0.462 & 14.0 & 0.481 & 3.5 & 1 \\
\hline RX CJ2217.7-3543 & A3854 & 221743.3 & -354334 & 0.1486 & 44 & 6.406 & 10.7 & 3.535 & 8.5 & 3.842 & 1.1 & 6 \\
\hline RX CJ2218.6-3853 & A3856 & 221840.2 & -385351 & 0.1411 & 10 & 7.132 & 10.2 & 3.516 & 9.0 & 3.781 & 1.3 & 6 \\
\hline RX CJ2234.5-3744 & A3888 & 223431.0 & -374406 & 0.1510 & 70 & 11.225 & 8.9 & 6.363 & 7.5 & 7.314 & 1.2 & 8 \\
\hline RX CJ2319.6-7313 & A3992 & 231941.8 & -731351 & 0.0984 & 3 & 3.993 & 17.9 & 0.937 & 7.5 & 1.030 & 1.9 & 3 \\
\hline
\end{tabular}

selection criterion, with an alternative selection scheme for the same clusters.

To illustrate the robustness of the approach in the presence of sample variance effects in the sample selection, we adopt for test purposes a different variant of the above selection scheme. We cut the bins in redshift space around the observed clusters, now using the midpoint rule on both sides of the bin. The resulting alternative bin boundaries are listed in Table 2 (this table also gives the luminosity values for the originally-chosen luminosity bins, defined for $H_{0}=50 \mathrm{~km} \mathrm{~s}^{-1} \mathrm{Mpc}^{-1}$ and a critical density universe). Note that now bins 8 and 9 begin at redshift $z=0$, since there are no lower redshift clusters with such a high X-ray luminosity in REFLEX.

For the extra, unobserved clusters in the bins, we again apply again the midpoint rule to exclude the regions containing the unobserved clusters. The cut-out zones in redshift space for this recipe are given in Table 2 . The cluster densities obtained with this second method are also given in Table 1 . We discuss the effect of the two different ways of defining the sample selection function in Sect. 7, where we use these data to construct the luminosity function for this sample.

\section{The sample}

In total 34 galaxy clusters were selected from the REFLEX catalogue for this study, as listed in Table 3. One of the selected objects, RX J1350.7-3343, was found to have a purely point source $\mathrm{X}$-ray emission from an AGN in the XMM-Newton images. In the RASS its X-ray emission was found to be significantly extended (visual inspection confirmed by the KS test). The origin of this extent is unclear, but could possibly be due to some attitude error in the data set that comes from different orbits. Such errors, if they occur at all in the RASS, must be very rare, since most of the known point like sources as stars and AGN do not feature such an extent. Therefore this object was removed from our sample and we have not included it in the above selection function construction. It is however listed in Table 3.

Table 3 gives information on the X-ray properties of the 33 clusters and the AGN X-ray source as determined from the ROSAT All-Sky Survey data. The columns of the table provide the following information: (1) the REFLEX name; (2) name given by Abell (1958) and Abell et al. (1989); (3) and (4) the right ascension and declination for the epoch J2000 in hours (degrees), minutes, and seconds; (5) the redshift; (6) the number of cluster galaxies from which the redshift has been determined; (7) and (8) the measured, unabsorbed X-ray flux, $F_{\mathrm{x}}$, in units of $10^{-12} \mathrm{erg} \mathrm{s}^{-1} \mathrm{~cm}^{-2}$ for the $0.1-2.4 \mathrm{keV}$ energy band and the fractional error in percent ${ }^{4}$; (9) the X-ray luminosity in units of $10^{44} \mathrm{erg} \mathrm{s}^{-1}$ in the rest frame 0.1 to $2.4 \mathrm{keV}$ band (uncorrected for missing flux); (10) the aperture radius in arcmin within which the X-ray count rate and flux were determined (the radius where the plateau value is reached in the cumulative count rate growth

\footnotetext{
4 The fluxes and luminosities quoted here are those measured in the ROSAT All-Sky Survey.
} 
Table 4. The additional clusters contained in the redshift-luminosity bins, which are not part of the observed cluster sample with more than 30 detected X-ray counts in the RASS and $N_{\mathrm{H}} \leq 6 \times 10^{20} \mathrm{~cm}^{-2}$.

\begin{tabular}{|c|c|c|c|c|c|c|c|c|c|c|c|c|}
\hline $\begin{array}{l}\text { Name } \\
(1)\end{array}$ & $\begin{array}{l}\text { Alt.name } \\
\text { (2) }\end{array}$ & $\begin{array}{r}\mathrm{RA}(2000) \\
(3) \\
\end{array}$ & $\begin{array}{r}\operatorname{Dec}(2000) \\
(4) \\
\end{array}$ & $\begin{array}{r}z \\
(5) \\
\end{array}$ & $\begin{array}{r}N_{\text {gal }} \cdot \\
(6)\end{array}$ & $\begin{array}{l}F_{\mathrm{x}} \\
(7) \\
\end{array}$ & $\begin{array}{r}\text { Error } \\
(8) \\
\end{array}$ & $\begin{array}{r}L_{\mathrm{x}} \\
(9) \\
\end{array}$ & $\begin{array}{r}R_{\text {ap }} \\
(10) \\
\end{array}$ & $\begin{array}{r}L_{\mathrm{x}}^{*} \\
(11) \\
\end{array}$ & $\begin{array}{r}N_{\mathrm{H}} \\
(12) \\
\end{array}$ & $\begin{array}{r}L_{\mathrm{x}}-\text { bin } \\
(13) \\
\end{array}$ \\
\hline RX CJ0028.6-2338 & A0042 & 002839.3 & -233814 & 0.1120 & 5 & 4.836 & 13.3 & 1.491 & 13.0 & 1.521 & 1.8 & 4 \\
\hline RX CJ0107.8-3643 & A2871 & 010749.1 & -364338 & 0.1186 & 19 & 3.557 & 13.6 & 1.229 & 12.0 & 1.254 & 1.9 & 4 \\
\hline RX CJ0108.9-1537 & A0151S & 010855.2 & -153744 & 0.0970 & 13 & 3.703 & 15.3 & 0.845 & 10.0 & 0.889 & 1.8 & 3 \\
\hline RX CJ0548.8-2154 & & 054850.4 & -215443 & 0.0928 & 9 & 3.977 & 12.3 & 0.825 & 11.5 & 0.859 & 3.0 & 3 \\
\hline RX CJ1038.4-2454 & & 103824.1 & -245410 & 0.1230 & 10 & 4.134 & 13.5 & 1.545 & 8.5 & 1.661 & 5.5 & 4 \\
\hline RX CJ1512.8-0128 & & 151251.0 & -012847 & 0.1223 & 2 & 3.354 & 18.5 & 1.238 & 5.5 & 1.423 & 5.2 & 4 \\
\hline RX CJ2220.5-3509 & A3866 & 222034.6 & -350953 & 0.1544 & 1 & 9.489 & 8.8 & 5.656 & 8.5 & 6.215 & 1.1 & 7 \\
\hline RX CJ2359.3-6042 & A4067 & 235919.2 & -604200 & 0.0989 & 30 & 4.544 & 20.5 & 1.080 & 9.5 & 1.149 & 2.4 & 3 \\
\hline
\end{tabular}

curve analysis); (11) the $0.1-2.4 \mathrm{keV}$ luminosity corrected for the estimated flux lost outside the measurement aperture (by extrapolating to a radius of 12 core radii by means of a $\beta$-model with $\beta=2 / 3$, see Böhringer et al. 2004, for more details); (12) the interstellar HI column density in units of $10^{20} \mathrm{~cm}^{-2}$ from Dickey \& Lockman (1990); and (13) the luminosity bin number to which the cluster belongs.

To provide a complete documentation, we also list in Table 4 those clusters which were scattered into the sample bins in luminosity-redshift space due to the reconstruction of our sample. They are not observed in this project but are statistically accounted for. This table is similar in structure to Table 3 and the parameter description is the same.

Three of the clusters had previous XMM-Newton observations in the archive. For A1689 (RX CJ1311.4-0120) and the A901/A902 cluster complex (RX CJ0956.4-1004), the exposure times were sufficient or longer than required for this study, and we thus could make use of the archive data. A3888 had only a very short exposure in the archive. Thus we complemented this observation by additional exposure time to bring the data to the same depth as for the other clusters. In our AO3 proposal we successfully requested the observation of 32 targets. Since about $37 \%$ of the observations suffered from severe contamination by solar flares for a substantial part of the observing time, we requested the reobservation of 3 clusters in $\mathrm{AO} 4$ and 9 further clusters in $\mathrm{AO} 5$. In this paper all the $\mathrm{AO} 4$ results are included. At the time of writing a large part, but not all, of the AO5 observations were completed and their data analysis is ongoing.

Table 5 provides an overview on the observation parameters and the data quality. In addition to the observation numbers, dates and nominal observation times, we give the mean LIVETIMES of the detectors chips. We only list the values for pn and MOS1, since the equivalent data for MOS2 are always very similar to those of MOS1. In the final columns we list the exposure times left for scientific analysis after a cleaning of the data for soft proton flares.

The cleaning done here, which is used to obtain a first overview on the data quality and to produce the image results shown below, is similar to that used in Pratt et al. (2007), where the data screening is optimized for the spectroscopic analysis. For a more detailed description of the screening we refer to that paper. In brief, we conduct a first data cleaning by means of a $3 \sigma$ clipping above the "quiet level" in the hard band light curves (12-14 keV for pn and 10-12 keV for MOS) in $100 \mathrm{~s}$ intervals, where the quiet level is characterized by a Gaussian distribution of the count rate at low count rate levels. In some cases where the observation is so disturbed that the Gaussian distribution is not easily established, we have used standard cut values, as noted in Table 5. The second screening is performed in a wider band
$(0.5-10 \mathrm{keV})$ in $10 \mathrm{~s}$ intervals. Most of the periods with high background are removed in the first cleaning step. The second stage mostly affects the flanks of the flares, and occasionally a flare which is very soft. Typically about $5-10 \%$ of the remaining data in flare-affected observations are removed in the second step. The cut values listed in Table 5 refer to the second wide band cleaning, and are given in units of counts in the total detector in $10 \mathrm{~s}$ intervals. We also remark on the effectiveness of this particular cleaning process for flagging data sets that are good, that have been cleaned by setting the cuts manually to a standard value, data which have an enhanced residual background, and data where one of the detectors is left with essentially no data.

\section{X-ray images of the sample clusters}

Figures 2 to 10 show images of the clusters in the $0.5-2 \mathrm{keV}$ band, an energy range which has an optimal signal-to-noise. The images are grouped by in bins of increasing luminosity. The images are produced from the cleaned event files, normalized by the exposure maps which include the vignetting correction, gaps and bad pixel information. The images of all detectors are combined with the pixel count rates of the two MOS detectors scaled to the pn sensitivity for a typical cluster spectrum. The combination is performed for the exposure maps and for the images separately, such that almost all gaps and bad pixel holes are filled by the information from at least one detector. The images are then smoothed by a Gaussian filter with a $\sigma$-width of $4 \operatorname{arcsec}$ (which is slightly less than the instrument PSF).

The colour scale of the images is scaled with a factor of $L_{\mathrm{X}}^{0.22}$, for the following reason. In the simple self similar picture of clusters (see e.g. Kaiser 1986) we expect the central intracluster plasma density to be roughly constant (ignoring the known deviations due to secular entropy modifications), and also the gas density profile as a function of the scaled radius, $r / r_{500}$ should be roughly the same (e.g. Arnaud et al. 2002). Since the surface brightness is proportional to the plasma density squared integrated along the line of sight through the cluster, the surface brightness then scales only with the line-of-sight extent of the cluster, that is with $r_{500}$. Taking $r_{500} \propto M^{1 / 3}$ and $L_{X} \propto M^{1.5}$ we obtain the above relation between X-ray luminosity and surface brightness. The scaling does not take into account the surface brightness dimming with redshift, however, although the redshift interval covered by these clusters is relatively small. The surface brightness contours used in the figures were not scaled but start at a fixed ratio to the typical background and increase in logarithmic steps (by a factor of $\sqrt{2}$ ).

With this scaling we readily recognize clusters with very dense cores (cooling cores) as those with very bright centres. 
Table 5. Overview of the XMM-Newton observation parameters of the cluster sample, up to and including AO4.

\begin{tabular}{|c|c|c|c|c|c|c|c|c|c|c|c|}
\hline Name & $\begin{array}{l}\text { Observation } \\
\text { number } \\
\text { (2) }\end{array}$ & $\begin{array}{r}\text { Date } \\
\text { (d.m.y) } \\
(3)\end{array}$ & $\begin{array}{r}\text { Nominal } \\
\text { exp. PN } \\
(4)\end{array}$ & $\begin{array}{r}\text { Nominal } \\
\text { exp. MOS1 } \\
(5)\end{array}$ & $\begin{array}{r}\text { Total } \\
\text { exp. PN } \\
(6)\end{array}$ & $\begin{array}{r}\text { Total } \\
\text { exp. MOS1 } \\
(7)\end{array}$ & $\begin{array}{r}\text { Cleaned } \\
\text { exp. PN } \\
(8)\end{array}$ & $\begin{array}{r}\text { Cleaned } \\
\text { exp. MOS } 1 \\
(9)\end{array}$ & $\begin{array}{l}\text { Cut } \\
\text { PN } \\
(10)\end{array}$ & $\begin{array}{r}\text { Cut } \\
\text { MOS1 } \\
(11)\end{array}$ & (12) \\
\hline RX CJ0003+0203 & 201900101 & 24.06 .04 & 23279 & 26667 & 20242 & 26223 & 19409 & 26002 & 102 & 37 & 1 \\
\hline RX CJ0006-3443a & 201900201 & 08.12 .04 & 17019 & 15454 & 8508 & 3109 & 1303 & 0 & 80 & 33 & 2 \\
\hline RX CJ0006-3443b & 201903801 & 13.05 .05 & 13836 & 17763 & 11872 & 17493 & 5852 & 12201 & 113 & 45 & 3 \\
\hline RX CJ0020-2542 & 201900301 & 26.05 .04 & 26379 & 29767 & 23001 & 29401 & 10732 & 15358 & 93 & 39 & 1 \\
\hline RX CJ0049-2931 & 201900401 & 04.12 .04 & 31333 & 34519 & 22992 & 33630 & 13304 & 19814 & 159 & 43 & 3 \\
\hline RX CJ0145-5300 & 201900501 & 12.11 .04 & 25764 & 27498 & 17997 & 27126 & 0 & 702 & 80 & 33 & 2 \\
\hline RX CJ0211-4017 & 201900601 & 27.12 .04 & 25240 & 29167 & 21829 & 28740 & 21741 & 28734 & 83 & 37 & 1 \\
\hline RX CJ0225-2928a & 201900701 & 06.07 .04 & 25301 & 29227 & 18205 & 0 & 4234 & 0 & 82 & 0 & 0 \\
\hline RX CJ0225-2928b & 302610601 & 27.01 .06 & 22440 & 26367 & 19197 & 26025 & 16519 & 20290 & 198 & 43 & 3 \\
\hline RX CJ0345-4112 & 201900801 & 05.03 .04 & 23279 & 26667 & 20541 & 26233 & 8154 & 17465 & 94 & 44 & 1 \\
\hline RX CJ0547-3152 & 201900901 & 07.03 .04 & 21679 & 25067 & 19144 & 24819 & 17604 & 23464 & 97 & 39 & 1 \\
\hline RX CJ0605-3518 & 201901001 & 29.10 .04 & 20940 & 26667 & 18136 & 26327 & 14756 & 20124 & 116 & 53 & 1 \\
\hline RX CJ0616-4748a & 201901101 & 26.04 .04 & 25379 & 27183 & 17523 & 26103 & 3689 & 6817 & 78 & 109 & 3 \\
\hline RX CJ0616-4748b & 302610401 & 05.01 .06 & 23940 & 27867 & 20493 & 27539 & 18719 & 22700 & 81 & 31 & 1 \\
\hline RX CJ0645-5413a & 201901201 & 07.05 .04 & 19874 & 18517 & 0 & 18328 & 0 & 11167 & 0 & 40 & 0 \\
\hline RX CJ0645-5413b & 201903401 & 12.06 .04 & 17279 & 20667 & 14936 & 20448 & 4304 & 6908 & 80 & 33 & 2 \\
\hline RX CJ0821+0112a & 201901301 & 3.10 .04 & 22001 & 15668 & 16285 & 7315 & 173 & 5921 & 80 & 33 & 2 \\
\hline RX CJ0821+0112b & 201903601 & 15.11 .04 & 7740 & 11667 & 6775 & 11545 & 6765 & 11469 & 73 & 30 & 1 \\
\hline RX CJ0956-1004 & 148170101 & 06.05 .03 & 94321 & 94333 & 3438 & 60448 & 3438 & 43202 & 52 & 27 & 1 \\
\hline RX CJ0958-1103a & 201901401 & 09.05 .04 & 12031 & 14950 & 10251 & 2038 & 1933 & 5000 & 76 & 50 & 3 \\
\hline RX CJ0958-1103b & 201903501 & 17.06 .04 & 11279 & 14667 & 9763 & 14433 & 4830 & 8588 & 99 & 43 & 1 \\
\hline RX CJ1044-0704 & 201901501 & 3.12 .04 & 25240 & 29167 & 21833 & 28827 & 18293 & 25712 & 109 & 45 & 1 \\
\hline RX CJ1141-1216 & 201901601 & 09.07 .04 & 32805 & 32274 & 24343 & 31653 & 21920 & 28274 & 76 & 30 & 1 \\
\hline RX CJ1236-3354a & 201901701 & 28.07 .04 & 19274 & 24268 & 15693 & 24026 & 260 & 6907 & 80 & 33 & 2 \\
\hline RX CJ1236-3354b & 201903701 & 30.12 .04 & 12140 & 16067 & 10562 & 15871 & 9448 & 13781 & 102 & 39 & 1 \\
\hline RX CJ1236-3354c & 302610701 & 20.01 .06 & 20940 & 24867 & 17974 & 24573 & 17870 & 24173 & 83 & 32 & 1 \\
\hline RX CJ1302-0230 & 201901801 & 22.06 .04 & 20479 & 25667 & 17844 & 25243 & 16443 & 24538 & 84 & 35 & 1 \\
\hline RX CJ1311-0120 & 093030101 & 24.12 .01 & 34798 & 39167 & 30682 & 38403 & 29224 & 36588 & 134 & 52 & 1 \\
\hline RX CJ1350-3343 & 201901901 & 15.02 .04 & 22879 & 26267 & 20171 & 25962 & 5294 & 14532 & 80 & 33 & 2 \\
\hline RX CJ1516+0005 & 201902001 & & 24240 & 28167 & 21209 & 27858 & 21058 & 26500 & 105 & 43 & 1 \\
\hline RX CJ1516-0056 & 201902101 & 03.08 .04 & 26240 & 30167 & 23030 & 29748 & 21750 & 29284 & 83 & 33 & 1 \\
\hline RX CJ2014-2430 & 201902201 & 08.10 .04 & 22740 & 26667 & 19531 & 26170 & 16041 & 24677 & 137 & 59 & 3 \\
\hline RX CJ2023-2056 & 201902301 & 06.04 .05 & 25740 & 29667 & 21070 & 29346 & 9205 & 17380 & 93 & 34 & 1 \\
\hline RX CJ2048-1750 & 201902401 & 13.05 .04 & 23279 & 26667 & 20166 & 26116 & 18728 & 25119 & 85 & 36 & 1 \\
\hline RX CJ2129-5048 & 201902501 & 16.10 .04 & 21740 & 25667 & 18827 & 25376 & 12508 & 23163 & 123 & 46 & 3 \\
\hline RX CJ2149-3041 & 201902601 & 29.11 .04 & 22740 & 26667 & 19698 & 26327 & 18019 & 25279 & 69 & 39 & 1 \\
\hline RX CJ2152-1942 & 201902701 & 28.10 .04 & 22740 & 26667 & 19674 & 26363 & 10962 & 21226 & 111 & 49 & 1 \\
\hline RX CJ2157-0747 & 201902801 & 11.05 .05 & 22740 & 24334 & 18491 & 17351 & 7387 & 10460 & 98 & 36 & 1 \\
\hline RX CJ2217-3543 & 201902901 & 12.05 .05 & 22741 & 26668 & 19899 & 26392 & 16767 & 23637 & 90 & 34 & 1 \\
\hline RX CJ2218-3853 & 201903001 & 24.10 .04 & 24340 & 28267 & 20918 & 27801 & 12328 & 22396 & 129 & 51 & 1 \\
\hline RX CJ2234-3744a & 201903101 & 10.11 .04 & 26540 & 30467 & 11728 & 30057 & 4808 & 59 & 80 & 33 & 2 \\
\hline RX CJ2234-3744b & 018741701 & 03.05 .01 & 4488 & 7114 & 4070 & 7050 & 3952 & 6836 & 135 & 53 & 1 \\
\hline RX CJ2319-7313a & 201903201 & 18.04 .04 & 24827 & 15681 & 8980 & 14987 & 0 & 279 & 999 & 565 & 3 \\
\hline RX CJ2319-7313b & 201903301 & 15.05 .04 & 7279 & 10667 & 6368 & 10543 & 6363 & 10277 & 83 & 35 & 1 \\
\hline
\end{tabular}

The nominal exposure times (Cols. 4 and 5) are obtained from the observation log browser of the XMM-Newton archive (http://xmm.vilspa.esa.es/external/xmm_obs_info/obs_view_frame.shtml) for each detecter. The total exposure times (Cols. 6 and 7) are the mean chip LIVETIMES read from the event file headers, and the cleaned times (Cols. 8 and 9) are obtained after the application of the two-step cleaning process described in the text. Columns 10 and 11 give the cut values for the second, wider band cleaning in units of cts per $10 \mathrm{~s}$. The flag indicates good cleaning (1), cleaning with standard cuts (2), imperfect cleaning with residual high background sufficient for the image analysis but not necessarily for spectroscopy (3), and cases where the exposure for one of the detectors has effectively been lost (0).

Clusters which barely reach green colours (displayed in the electronic version of the paper) feature a very low surface brightness, indicating that these clusters are most probably dynamically young.

\section{Remarks on some clusters}

There will be at least one dedicated publication addressing in detail the morphology of the clusters in this sample. Here we briefly comment on some of the peculiar clusters. There are 2 clusters with multiple components, 3 clusters with a complex, diffuse, low surface brightness appearance, and one cluster where the data are still sparse.

RX CJ2152.2-1942 (Fig. 4) was selected as the fainter southern component of this bimodal cluster. In the ROSAT AllSky Survey the two emission regions appear almost distinct and the system was therefore split into two clusters (two separate dark matter halos) in the REFLEX catalogue. In the survey selection only the southern component should be counted. The deeper XMM-Newton exposure now reveals that the two systems are interacting. The total system is catalogued in the optical as A2384 by Abell (1958). 


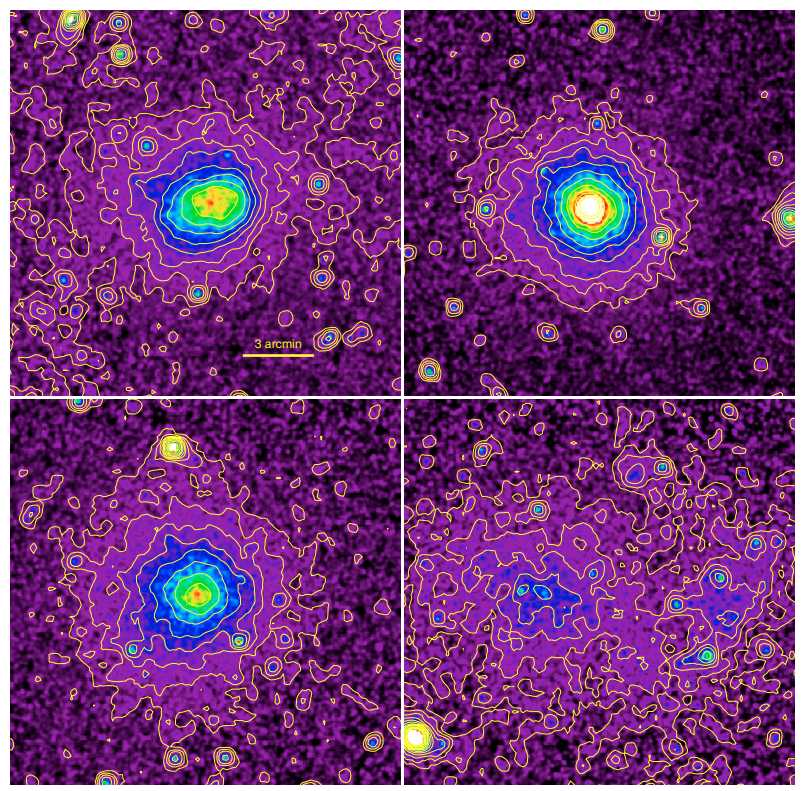

Fig. 2. Combined XMM-Newton MOS/pn $0.5-2 \mathrm{keV}$ images of the clusters in luminosity bin $1\left(4.07-5.5 \times 10^{43} \mathrm{erg} \mathrm{s}^{-1}\right)$, RX CJ0345.7-4112 (S384, upper left), RX CJ0225.1-2928 (upper right), RX CJ2023.0-2056 (S868, lower left), and RX CJ2157.4-0747 (A2399, lower right). The images have been corrected for vignetting and detector gaps, and the surface brightness of the combined image has been normalized to that of the pn detector. The background (not subtracted) is typically at a level of $4-4.5 \times 10^{-3} \mathrm{cts} \mathrm{s}^{-1} \mathrm{arcmin}^{-2}$. The scale of the image is marked by a 3 arcmin long bar. The contours start at a surface brightness of $7.9 \times 10^{-3} \mathrm{cts} \mathrm{s}^{-1} \operatorname{arcmin}^{-2}$ and increase in steps of $\sqrt{2}$.

RX CJ0956.4-1004 (Fig. 9), also known as A901a, A901b, and A902, is a system of several diffuse and point-like X-ray sources. In the ROSAT All-Sky Survey we observed a complex emission region that was catalogued as one object. For our analysis we have used the archival XMM-Newton observation. The nominal observing time of this observation was very long, $\sim 94 \mathrm{ks}$, but only half of the MOS observing time is useful due to a series of strong flares, and the pn detector was closed during the observation.

Gray et al. (2002) find three major mass concentrations, A901a, A901b, and A902, in their lensing analysis, and call the structure a supercluster at redshift $z=0.16$. Only A901b shows the extended, but compact, $\mathrm{X}$-ray emission expected from a well evolved rich X-ray luminous cluster, as noted previously from the ROSAT HRI observation by Schindler (2000). The $\mathrm{X}$-ray emission from A901a is dominated by a very strong point source, associated with a faint galaxy. There is definitely also extended emission associated with this mass component. The extended X-ray emission is centered on the central dominant elliptical of A901a in the west of the X-ray point source and very diffuse low surface brightness emission is observed on larger scale. The third mass component A902 is also associated with very diffuse low surface brightness emission, which indicates a dynamically very young galaxy cluster structure. The extended X-ray emission around A901a and A902 was not noted in the ROSAT HRI study by Schindler (2000), which involved much fewer photons. More details on the morphology of this cluster will be described in a forthcoming paper from our collaboration.

RX CJ2157.4-0747 (Fig. 2), A2399, is a bimodal system with very diffuse, low surface brightness X-ray emission. Like the following two clusters, this is most probably a dynamically
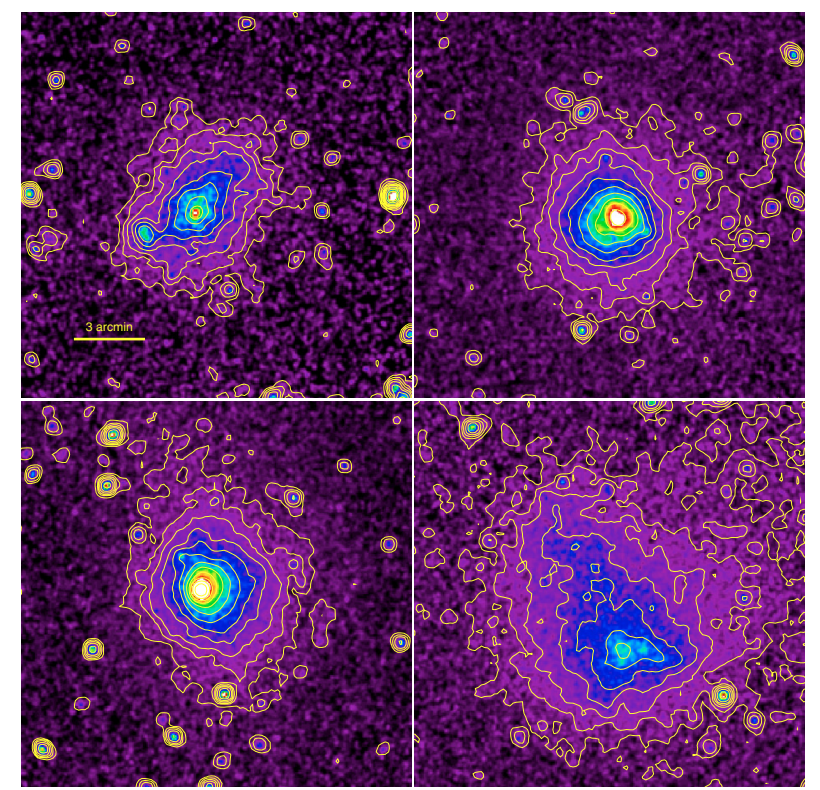

Fig. 3. Combined XMM-Newton MOS/pn $0.5-2 \mathrm{keV}$ images of the clusters in luminosity bin $2\left(5.5-7.8 \times 10^{43} \mathrm{erg} \mathrm{s}^{-1}\right)$, RX CJ0821.8+0112 (A653, upper left), RXCJ1236.7-3354 (S700, upper right), RXCJ1302.8-0230 (A1663, lower left), and RXCJ2129.8-5048 (A3771, lower right). The details are the same as in Fig. 2.

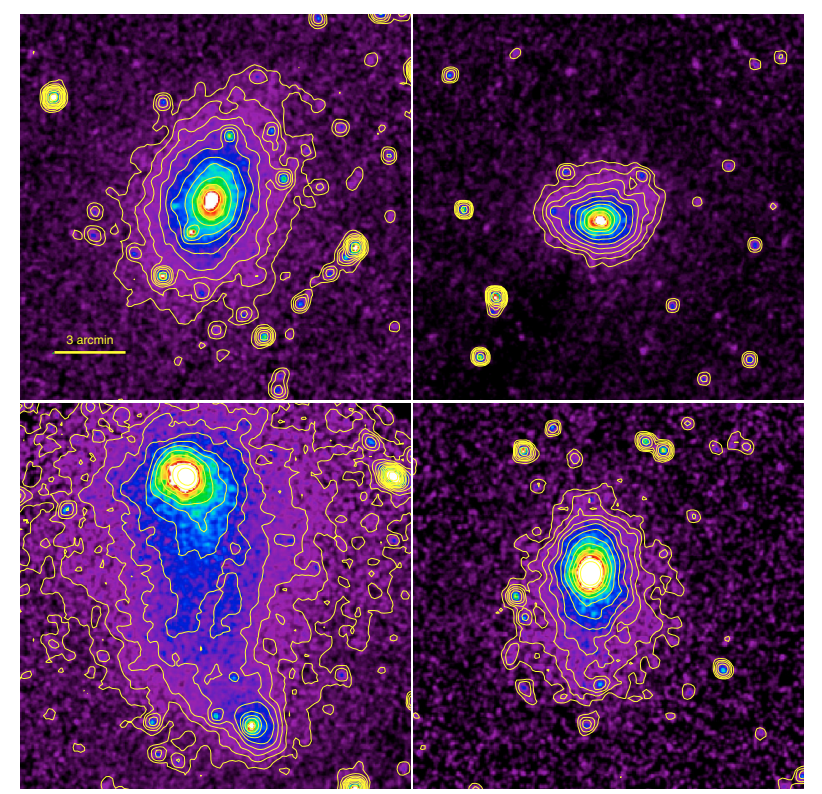

Fig. 4. Combined XMM-Newton MOS/pn $0.5-2 \mathrm{keV}$ images of the clusters in luminosity bin $3\left(0.78-1.13 \times 10^{44} \mathrm{erg} \mathrm{s}^{-1}\right)$, RX CJ0003.8+0203 (A2700, upper left), RXCJ0211.4-4017 (A2984, upper right), RXCJ2152.2-1942 (A2384B, lower left), and RXCJ2319.6-7313 (A3992, lower right). The details are the same as in Fig. 2.

young object in formation without a significant preexisting cluster core.

RX CJ2129.8-5048 (Fig. 3), A3771, is another low surface brightness cluster, which is dynamically young, but does not feature a multi-component configuration.

RX CJ2048.1-1750 (Fig. 7), A2328, is similar in its morphology to the previous cluster, but is more luminous and thus massive. In addition it features two smaller possibly infalling systems at its outskirts. 


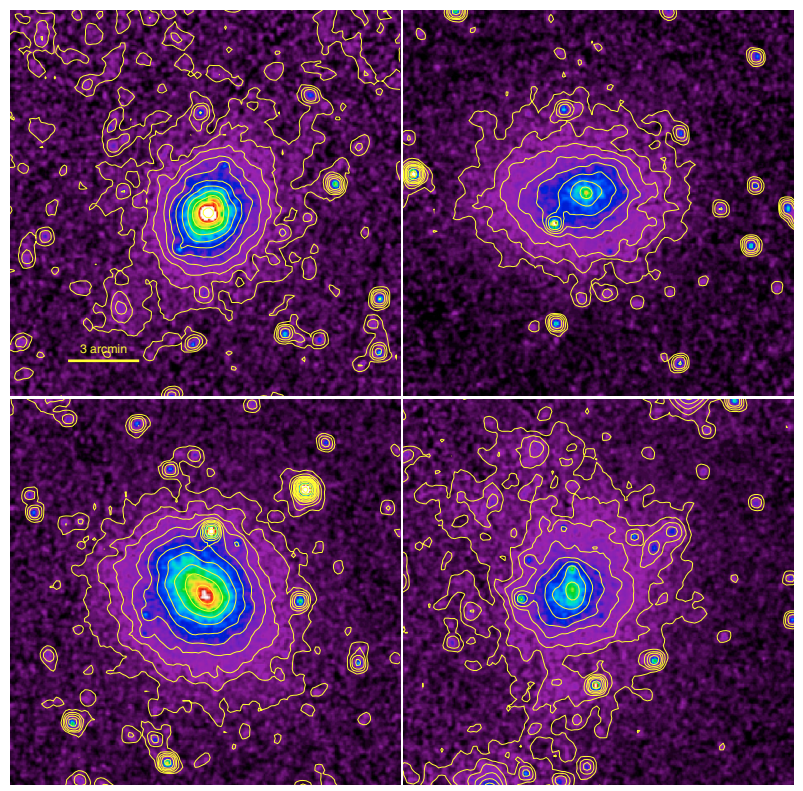

Fig. 5. Combined XMM-Newton MOS/pn 0.5-2 keV images of the clusters in luminosity bin $4\left(1.13-1.71 \times 10^{44} \mathrm{erg} \mathrm{s}^{-1}\right)$, RX CJ0049.4-2931 (S84, upper left), RX CJ0616.8-4748 (upper right), RXCJ1516.3+0005 (A2050, lower left), and RXCJ1516.5-0056 (A2051, lower right). The details are the same as in Fig. 2.

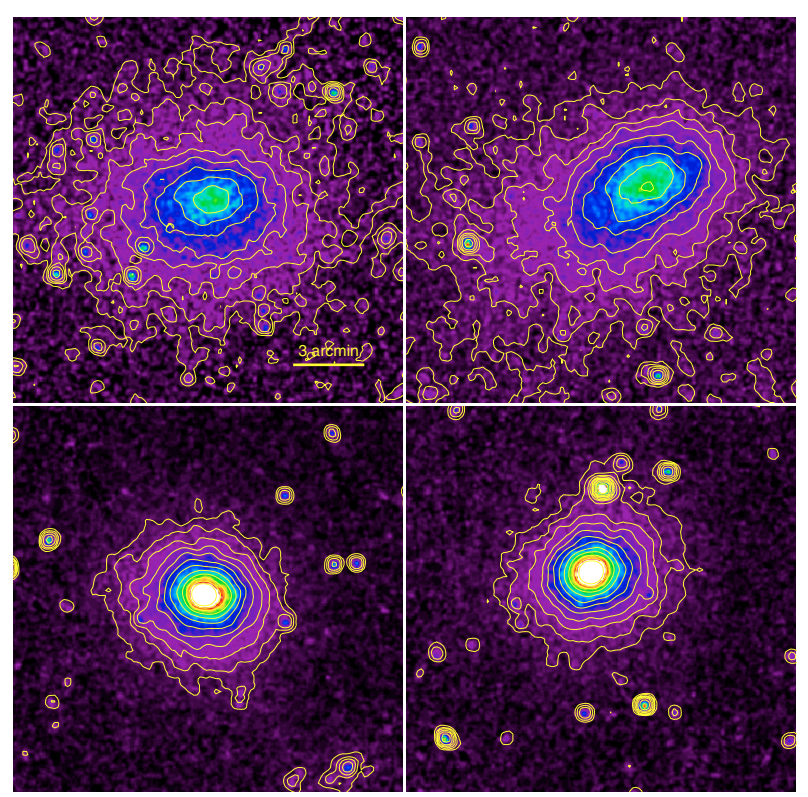

Fig. 6. Combined XMM-Newton MOS/pn 0.5-2 keV images of the clusters in luminosity bin $5\left(1.71-2.88 \times 10^{44} \mathrm{erg} \mathrm{s}^{-1}\right)$, RX CJ0006.03443 (A2721, upper left), RX CJ0145.0-5300 (A2941, upper right), RX CJ1141.4-1216 (A1348, lower left), and RX CJ2149.1-3041 (A3814, lower right).The image of RX CJ0145.0-5300 was produced from AO5 data, since none of the previous observations left enough photons after cleaning for a decend image.

RX CJ0145.0-5300 (Fig. 6), A2941, has insufficient data even for the production of a decent X-ray image. After flare cleaning there is no useful pn time and only a few hundred seconds of MOS exposure are left, from which the image has been made. The observation of this cluster is rescheduled in AO5.

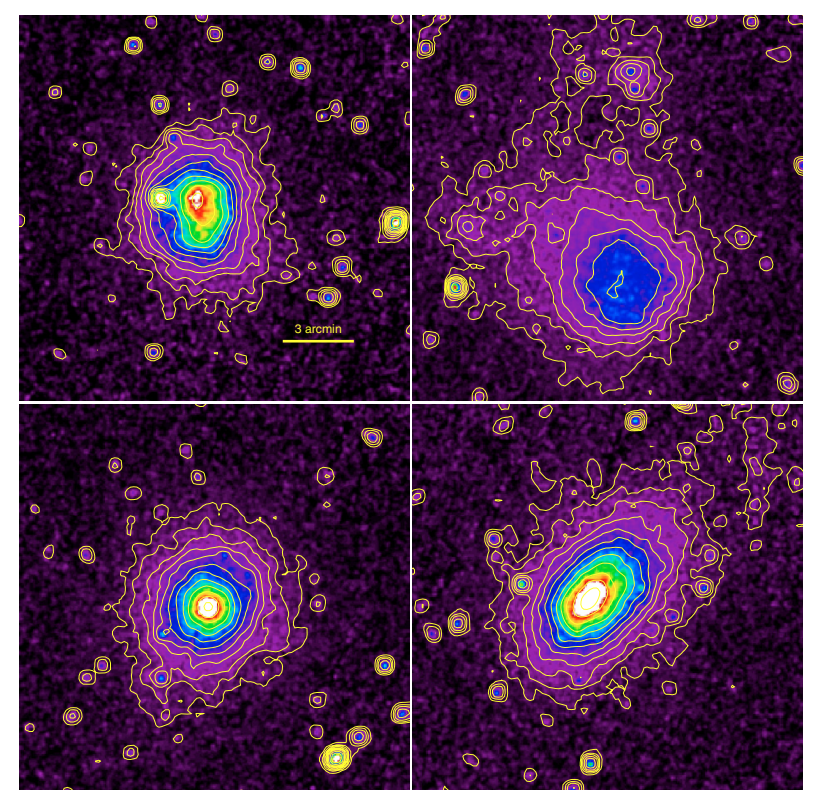

Fig. 7. Combined XMM-Newton MOS/pn $0.5-2 \mathrm{keV}$ images of the clusters in luminosity bin $6\left(2.88-4.10 \times 10^{44} \mathrm{erg} \mathrm{s}^{-1}\right)$, RX CJ0020.7-2542 (A22, upper left), RX CJ2048.1-1750 (A2328, upper right), RXCJ2217.7-3543 (A3854, lower left), and RX CJ2218.6-3853 (A3856, lower right). The details are the same as in Fig. 2.

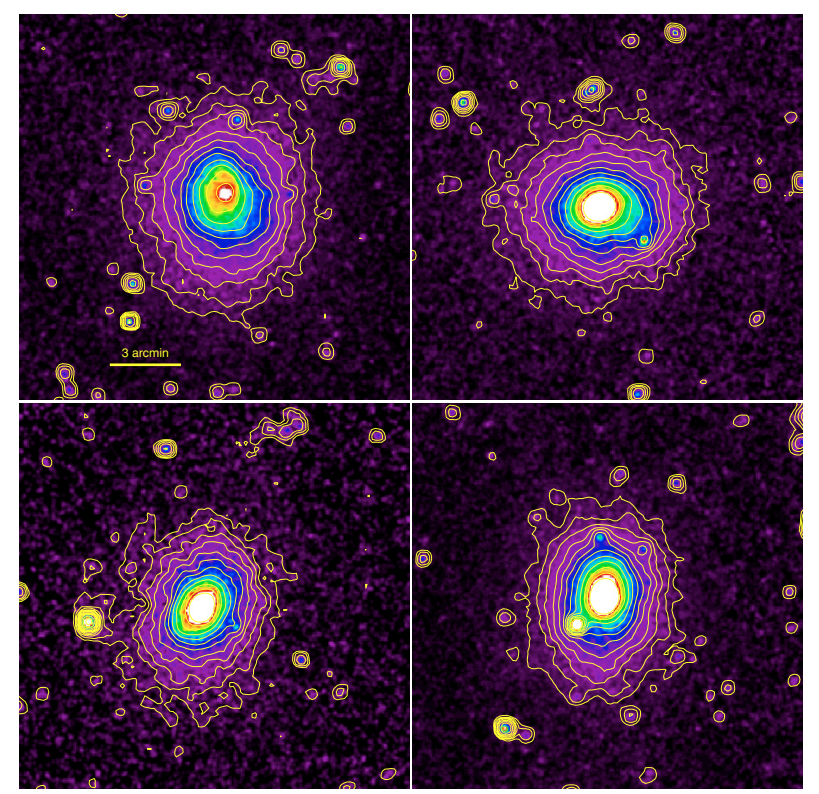

Fig. 8. Combined XMM-Newton MOS/pn 0.5-2 keV images of the clusters in luminosity bin $7\left(4.10-5.90 \times 10^{44} \mathrm{erg} \mathrm{s}^{-1}\right)$, RX CJ0547.6-3152 (A3364, upper left), RXCJ0605.8-3518 (A3378, upper right), RXCJ0958.3-1103 (A907, lower left), and RX CJ1044.5-0704 (A1084, lower right). The details are the same as in Fig. 2.

\section{Comparison between ROSAT All-Sky Survey and XMM-Newton fluxes}

Using the present XMM-Newton observations we measured the fluxes from the galaxy clusters in the $0.5-2 \mathrm{keV}$ band, and compared the results to the previous ROSAT All-Sky Survey (RASS) observations. In the RASS the count rates from which the fluxes are derived were determined by the growth curve analysis 


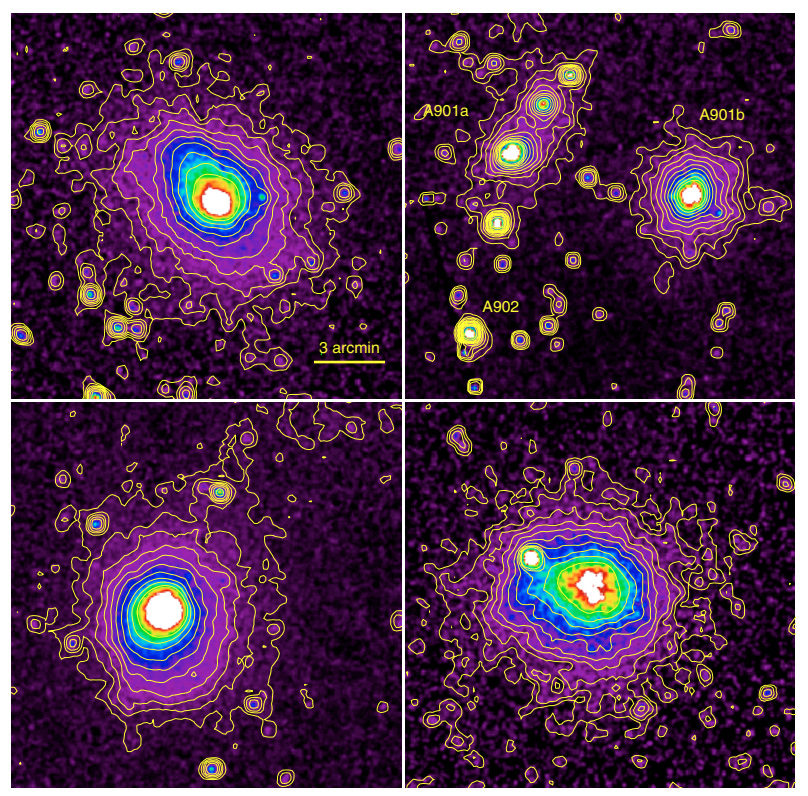

Fig. 9. Combined XMM-Newton MOS/pn $0.5-2 \mathrm{keV}$ images of the clusters in luminosity bin $8\left(5.90-11.9 \times 10^{44} \mathrm{erg} \mathrm{s}^{-1}\right)$, RX CJ0645.4-5413 (A3404, upper left), RX CJ0956.4-1004 (A901, upper right), RXCJ2014.8-2430 (lower left), and RXCJ2234.5-3744 (A3888, lower right). The details are the same as in Fig. 2. For the image of RX CJ0956.4-1004 only data from the two MOS detectors have been used, since the pn detector was closed during the observation.

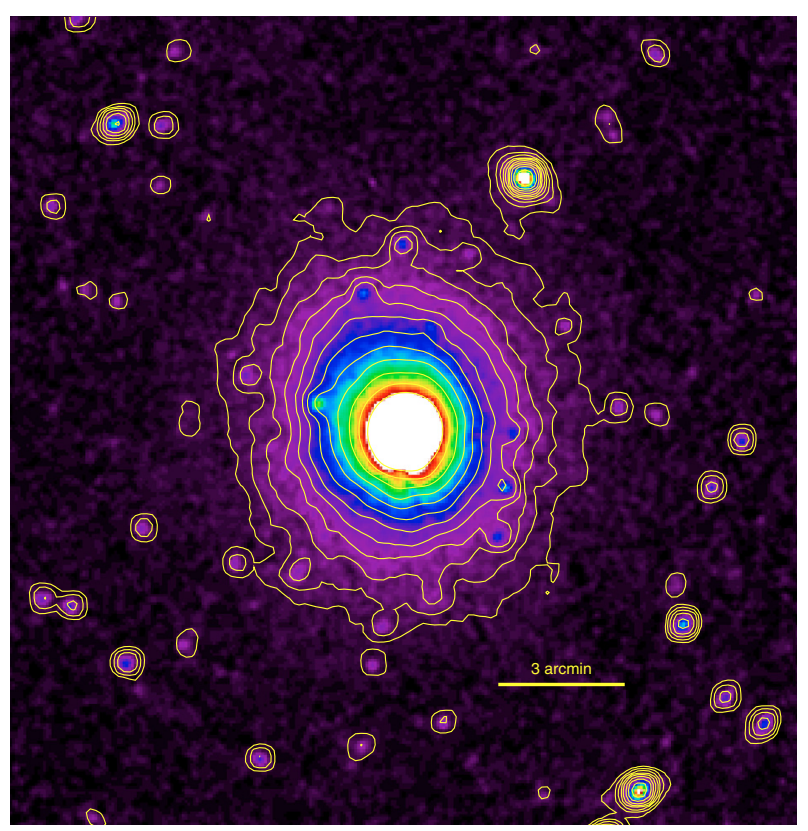

Fig. 10. Combined XMM-Newton MOS/pn $0.5-2 \mathrm{keV}$ images of the clusters in luminosity bin $9\left(11.9-20 \times 10^{44} \mathrm{erg} \mathrm{s}^{-1}\right)$. The details are the same as in Fig. 2.

technique described in Böhringer et al. (2000). Here we use a similar approach.

We first construct cluster images for each detector. We then integrate the counts in concentric rings, weighting each image pixel by the vignetting corrected exposure maps. We excise all bad pixels and pixels which fall into gaps or low exposure regions near gaps, and correct for the area lost in the ring. To estimate the background contribution we use the background data provided by Read and Ponman (Read \& Ponman 2003)

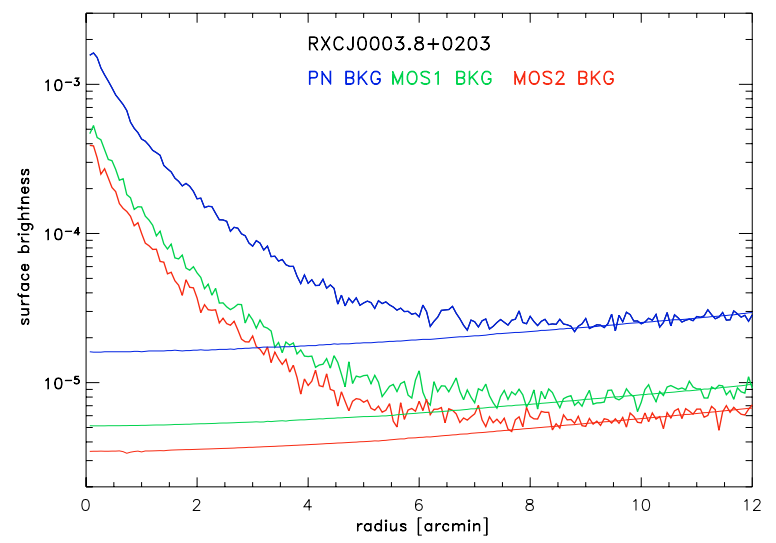

Fig. 11. $[0.5-2 \mathrm{keV}]$ surface brightness profile of the cluster RXCJ0003.8+0203, for all three detectors, in units of counts per $4 \times 4 \operatorname{arcsec}^{2}$ pixel s${ }^{-1}$ (upper curves), plotted with the scaled, modelled background surface brightness (lower curves). The MOS2 surface brightness profile has been multiplied by a factor of 0.7 for better visibility. The background surface brightness is increasing with radius because the background is vignetting corrected, which overweights the particle background in the outer regions. No significant cluster emission is seen for this target at radii outside about 9 arcmin.

with the same cleaning as applied by Pratt et al. (2007), recast onto the same sky position and orientation as the target fields. In these data sets X-ray sources have been removed and the images produced from the data sets feature depressions in these removal zones. We therefore apply a model fit to the background by means of the SAS task esplinemap with the parameter fitmethod $=$ model . We compare the model background surface brightness distribution to the target data in the same outer region (where we have insignificant cluster contribution to the X-ray image) and scale the background to the image surface brightness in this region where the profiles have the same shape. This scaled background is then subtracted from the cluster profile. An example of a cluster profile and the scaled background is shown in Fig. 11. We have tested the validity of this procedure by checking the change of the results as a function of the radius limit outside which the data are used for the renormalization and find very little change $(\leq 1 \%)$ for limiting radii $\geq 9$ or 10 arcmin, depending on the shape of the cluster.

To account for the point source contribution to a cluster's X-ray emission we have also produced "cleaned" images in the following way. We have run the $S A S$ source detection procedure ewavelet to localize point sources. Since also the cluster centers and substructure are usually recognized as X-ray sources by ewavelet we have removed the detected sources through visual inspection, retaining all diffuse cluster emission including substructure and central cusps. We used the radii of the ewavelet algorithm in SAS as excision radii, in a first attempt to exclude the point sources. This radius is increased after visual inspection for the brighter sources which are not completely removed. The same regions are excised in the exposure maps. We use these and the uncleaned images to measure the cluster and the total flux in the cluster region, respectively.

An integration of the surface brightness profiles times the area of the rings gives the count rate growth curves as shown in Fig. 12. These level off at large radii. Fluxes are derived from these count rates by means of count rate to flux conversion factors determined using XSPEC software ${ }^{5}$. To determine these conversion factors a spectral model has to be defined in

\footnotetext{
${ }^{5}$ http://heasarc.gsfc.nasa.gov/docs/xanadu/xspec
} 


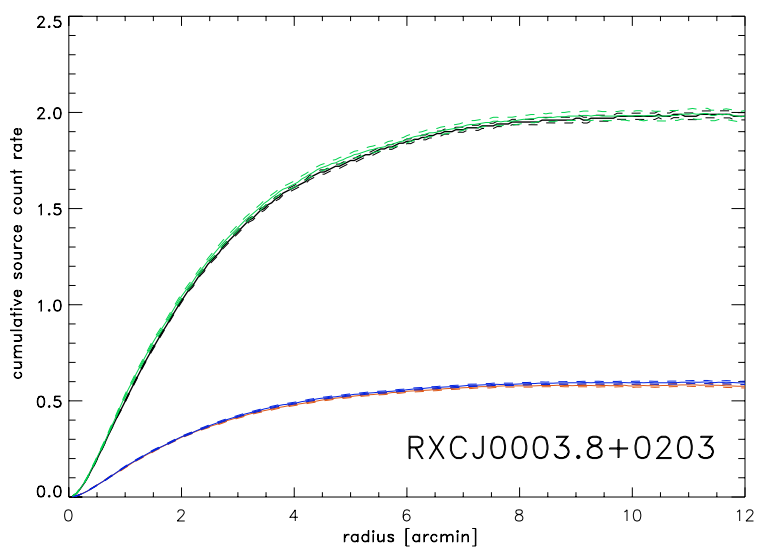

Fig. 12. Cumulative, background subtracted $[0.5-2 \mathrm{keV}]$ count rates ("growth curves") of the cluster RX CJ0003.8+0203 as a function of the cluster radius. The black upper curve (with error corridors) refers to the pn results, and the two lower curves with their errors corridors correspond to the two MOS results, respectively. The green, upper curve is the MOS1 growth curve scaled to the sensitivity of the pn. The curves reach a flat plateau outside a radius of about 9 arcmin.

XSPEC. For 15 of the clusters, we use an absorbed MEKAL model ${ }^{6}$ with a temperature as measured by a single temperature fit to the data in the radial region 0.1 to $0.4 r_{200}$ according to the analysis performed by Pratt et al. (2007). Accurate temperature measurements of this kind are not yet available for some clusters. For these remaining clusters we use a temperature estimate from the $L_{\mathrm{X}}$-temperature relation defined by Eq. (1), with the interstellar column densities listed in Table 3 and metallicities of 0.3 solar. The uncertainty in the flux conversion factor, even in the case of a factor 2 difference in the temperature estimate, is never larger than 3\%, and in most cases is much less. The error in the measured flux accounts for the Poisson error of the source counts as well as the photon statistical error in the background subtraction. More precise values for the fluxes and luminosities will be reported in a later paper when all the data are at hand and have been reduced. For these final results we will also consider the additional correction for the temperature variation with radius.

Here we are primarily interested in assessing the reliability with which the cluster fluxes have been determined in the RASS data. Therefore we take the fluxes determined by Böhringer et al. (2004), which were obtained with the growth curve analysis method for a certain aperture radius (before the correction to total fluxes). We apply the growth curve technique to the XMM-Newton data out to the same aperture radius, separately for the three detectors (since we have sufficiently good statistics), and compare all four results in Figs. 13 and 14. For the XMM-Newton data we can reliably determine the growth curve flux only to a maximum radius of 12 arcmin because of the XMM-Newton field-of-view, while for 7 clusters in the sample the RASS measurement aperture is larger than this (see Table 3). Therefore we have estimated an upper limit on the extra flux that might be seen in the RASS at the larger radii. It is smaller than $2 \%$ for three of the clusters (RX CJ0605-3518, RX CJ0645-5413, RX CJ0956-1004), smaller than $6 \%$ for three further clusters (RX CJ0457-3152, RX CJ1516-0056, RX CJ2157-0747) and larger by $\leq 12 \%$ for RX CJ0616-4748. This is, apart from the last case, smaller than

\footnotetext{
${ }^{6}$ http://heasarc.gsfc.nasa.gov/docs/xanadu/xspec/ manual/XSmodelMekal.html
}
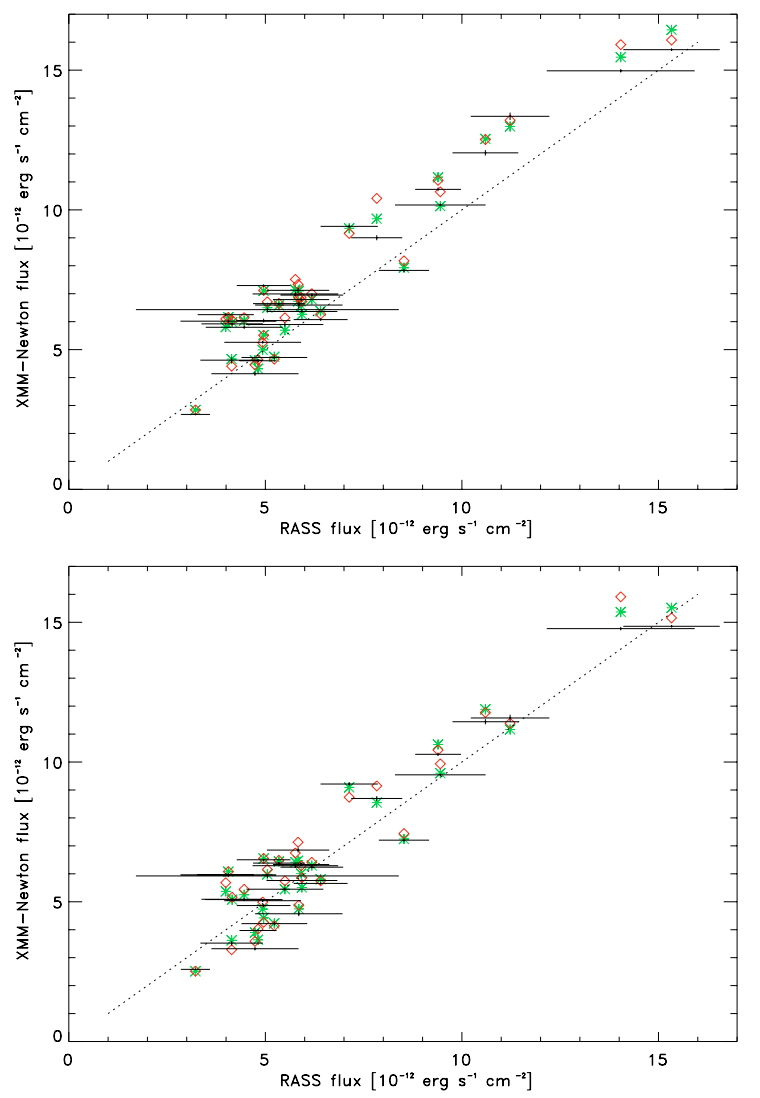

Fig. 13. Comparison of REFLEX clusters fluxes determined from the ROSAT All-Sky Survey and the fluxes obtained from the three detectors of XMM-Newton: black crosses (pn), diamonds (MOS1), green stars (MOS2). The upper panel shows the results for the total XMM-Newton cluster fluxes, while the lower panel shows the XMM-Newton cluster fluxes with point source contamination removed. Error bars are only shown at the location of the pn data points. The vertical error bars are generally smaller than the plotted symbols.

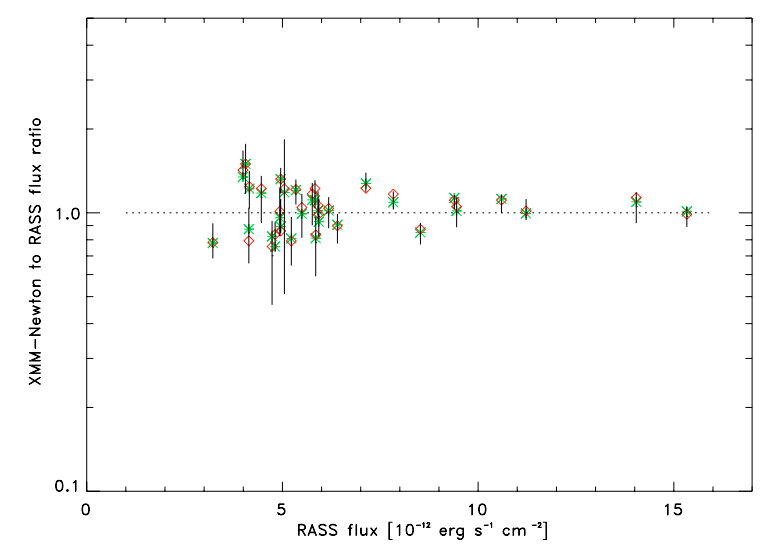

Fig. 14. Ratio of the XMM/Newton observations (contaminating point sources subtracted) to the RASS observations as a function of RASS flux. The symbols have the same meaning as in Fig. 13.

the quoted $1 \sigma$ uncertainty. Note also that the angular resolution of the RASS is much worse (more than one arcmin) than that of the XMM-Newton observations and therefore the unsharp apertures are not exactly the same.

There is a good agreement within the uncertainties of the RASS flux determination. In Fig. 13 we compare both the XMM-Newton fluxes and the point source subtracted 


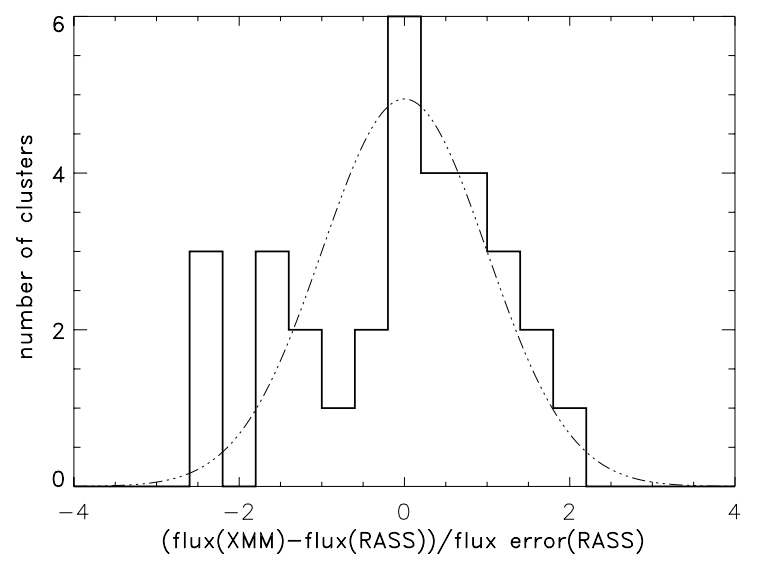

Fig. 15. Ratio of the measured flux difference between XMM-Newton (average of all three detectors) and RASS to the estimated error of the RASS. Also shown is a Gaussian distribution with $\sigma=1$, normalized to the total number of clusters. The good agreement of the two curves (except for the three outliers at low XMM-Newton flux) show that the estimated flux errors of the REFLEX sample are precise and reliable. The symbols have the same meaning as in Fig. 13.

XMM-Newton fluxes, with the RASS data, for the same detection aperture. While the unsubtracted XMM-Newton fluxes are on average about $10 \%$ higher than the RASS fluxes (the intercalibration of the two instruments is not known to much better than about $5 \%$ ), the point source corrected fluxes are in the average only about $2 \%$ different. Figure 14 shows that the deviations in the RASS fluxes decreases with the flux level as would be expected. Thus we conclude that the REFLEX catalogue contains very reliable flux estimates in spite of the very low number of photons available. These good results are made possible by the very low X-ray background of the RASS. This is also reflected by the fact that with the present XMM-Newton data, even with the superb photon statistics, we cannot extend the flux measurement to much larger aperture radii than was done with the RASS data.

Almost as important as a good flux measurement for the REFLEX catalogue are good estimates for the flux uncertainties. The latter parameter is also an important input into the construction of a precise cosmological model test (e.g. in analogy to Stanek et al. 2006). If Fig. 15 we test the reliability of this parameter, where we compare the flux uncertainty estimates for the RASS results with the deviations between RASS and XMM-Newton fluxes (assuming, to first order, that the uncertainties in the XMM-Newton fluxes are insignificant). Again we find excellent agreement.

Finally, Fig. 16 provides the statistics of the point source contribution to the total cluster flux in the REXCESS sample. We have no clusters where a point source is dominant. Excluding the complex supercluster A901/902, the unsufficient data set of RX CJ0145.0-5300, and the AGN RXJ1350.7-3343 from the analysis, we find a mean flux contamination of only $\sim 11 \%$, and none of the clusters has a larger contamination than $26 \%$ by point sources, as already expected from general tests on the REFLEX data.

\section{Construction of distribution functions}

As the survey volume of the cluster sample is well defined, we can construct absolute distribution functions for properties of these galaxy clusters. As an example we reconstruct the X-ray luminosity function of this sample by means of the cluster

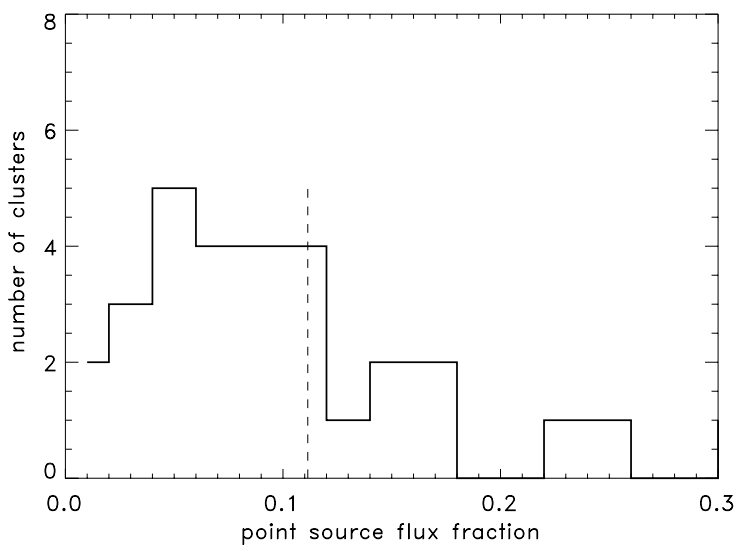

Fig. 16. Statistics of the point source contribution to the cluster fluxes for the sample (excluding 1 complex supercluster, one insufficient data set, and the AGN, RXJ1350.7-3343). The fraction is calculated by dividing the point source contribution by the point source subtracted cluster flux. The flux contamination is in all cases smaller than $26 \%$ and is on average about $11 \%$.

densities derived in Sect. 2. Figure 17 shows the results for the luminosity function using both selection schemes outlined in Sect. 2. The results are compared to the REFLEX X-ray luminosity function derived in Böhringer et al. (2002), which provides the luminosity function for the REFLEX sample as observed without an evolution correction. There is a good agreement between the results of the subsample and the total survey sample, with the largest deviation in the two highest luminosity bins (although these deviations are within the errors). This effect is due to the deficiency in the Southern sky of luminous $\mathrm{X}$-ray clusters in the nearby Universe. The effect is illustrated by the difference of the two selection recipe methods: if we use the nearest neighbour boundaries, the last two bins extend to $z=0$, resulting in a smaller cluster density in better agreement with the overall REFLEX result. This shows that the difference of the density of the most massive clusters in REFLEX and in the subsample is due to a real density variation in the Universe. The most massive clusters are highly biased and unevenly distributed in the REFLEX volume. The general good agreement of the two methods of the selection function construction shows that our approach is robust.

In this analysis we have assumed that the uncertainty in the luminosity measurement in the REXCESS sample is negligible. This uncertainty was taken into account in the analysis of the RASS data, where the uncertainties are larger (Böhringer et al. 2002).

\section{Summary and conclusions}

We have described a sample of 33 galaxy clusters which are selected purely on the basis of their X-ray luminosity in nearby redshift shells. The sample is therefore representative of an unbiased, X-ray luminosity or flux selected subset of the galaxy cluster population ${ }^{7}$. The study is designed to make the best use of the XMM-Newton observatory to provide comprehensive galaxy cluster structure statistics, and representative scaling relations.

The results show that the observational results from the REFLEX Cluster Survey in the RASS are recovered with excellent agreement, except for one REFLEX cluster candidate which

\footnotetext{
7 A flux limit is an effective luminosity selection for each redshift shell.
} 

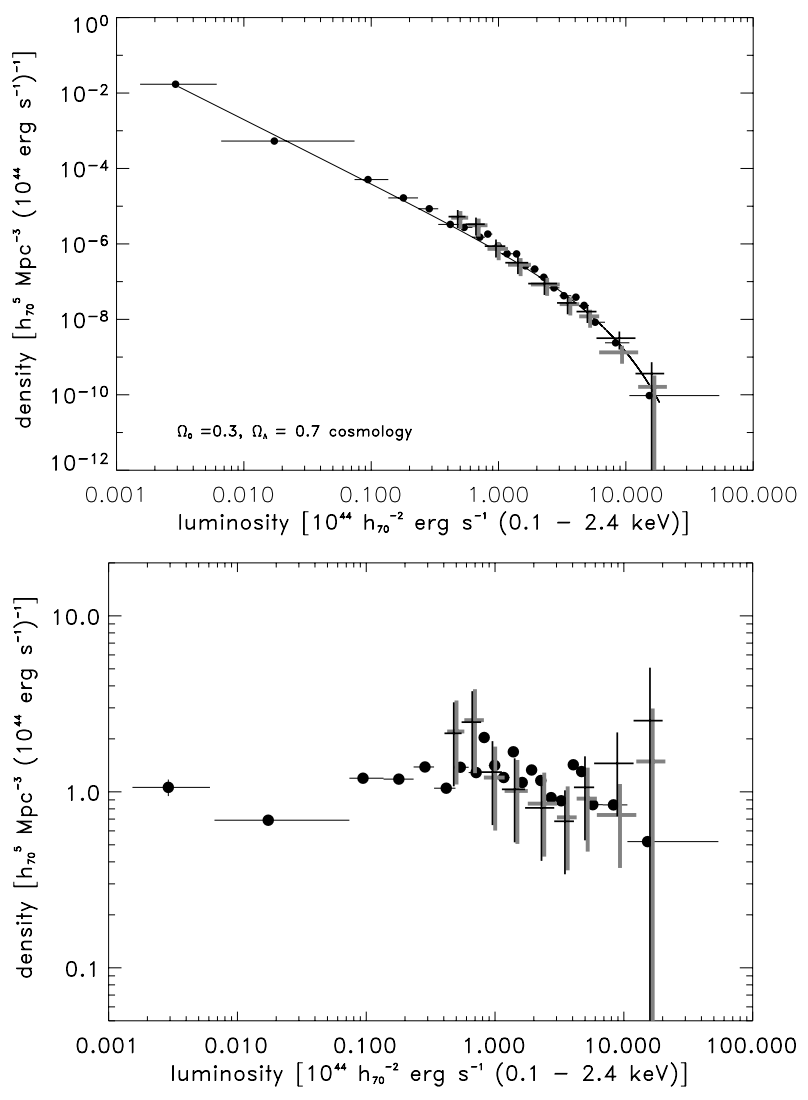

Fig. 17. X-ray luminosity function for the REFLEX sample and for the REXCESS subsample; the latter has been constructed by the two different methods explained in the text. Solid dots with error bars refer to the REFLEX function, black crosses mark the function constructed with the original selection function, and grey crosses the function obtained with the alternative method (Sect. 2). The lower panel shows the same data divided by the Schechter function fit to the REFLEX X-ray luminosity function derived in Böhringer et al. (2002).

was found here to be an X-ray AGN. The redetermined X-ray fluxes agree within a few percent and the flux errors are also in good agreement.

A detailed description of the cluster sample selection function allows us to determine the space density of any subsample with certain properties. We demonstrated how the selection function can be applied for the evaluation of the distribution function of cluster properties for the case of the X-ray luminosity function. The majority of the clusters show a roughly regular appearance, very often with elongations. Only a few clusters feature several components or peaks in the X-ray surface brightness distribution. Some clusters have a somewhat diffuse, low surface brightness structure. There is no dramatic merging cluster among the objects in the sample, indicating that these systems are probably rare in the general cluster population at $z \leq 0.2$.

This paper introduces the survey project, for which a series of papers covering extensive cluster structure analyses is in progress.

Acknowledgements. We thank our colleague and coauthor Peter Schuecker, who is sadly no longer with us, for many years of friendship and fruitful collaboration. The paper is based on observations obtained with XMM-Newton, an ESA science mission with instruments and contributions directly funded by ESA Member States and the USA (NASA). The XMM-Newton project is supported by the Bundesministerium für Bildung und Forschung, Deutsches Zentrum für Luft und Raumfahrt (BMBF/DLR), the Max-Planck Society and the HaidenhainStiftung. G.W.P. acknowledges support from DfG Transregio Programme TR33; D.P. acknowledges support by the German BMBF through the Verbundforschung under grant no. 50 OR 0405; A.K.R. acknowledges partial financial support from research grants MIRG-CT-2004-513676 and NASA LTSA 8390; K.P. acknowledges support from Instrument Center for Danish Astrophysics (The Dark Cosmology Centre is funded by the Danish National Research Foundation); H.Q. thanks the FONDAP Centro de Astrofisica for partial support. T.H.R. acknowledges support from the Deutsche Forschungsgemeinschaft through Emmy Noether Research Grant RE 1462 and from the German BMBF through the Verbundforschung under grant no. 50 OR 0601.

\section{References}

Arnaud, M., Aghanim, N., \& Neumann, D. M. 2002, A\&A, 389, 1 Arnaud, M., Pointecouteau, E., \& Pratt, G. W. 2005, A\&A, 441, 893 Abell, G. O. 1958, ApJS, 3, 211

Abell, G. O., Corwin, H. G., \& Olowin, R. P. 1989, ApJS, 70, 1

Allen, S. W., Schmidt, R. W., Ebeling, H., Fabian, A. C., \& van Speybroeck, L. 2004, MNRAS, 353, 457

Böhringer, H., Voges, W., Huchra, J. P., et al. 2000, ApJS, 129, 435

Böhringer, H., Schuecker, P., Guzzo, L., et al. 2001, A\&A, 369, 826

Böhringer, H., Collins, C. A., Guzzo, L., et al. 2002, ApJ, 566, 93

Böhringer, H., Schuecker, P., Guzzo, L., et al. 2004, A\&A, 425, 367

Borgani, S., \& Guzzo, L. 2001, Nature, 409, 39

Borgani, S., Governato, F., Wadsley, J., et al. 2001, ApJ, 559, L71

Borgani, S., Murante, G., Springel, V., et al. 2004, MNRAS, 348, 1078

Borgani, S., Finoguenov, A., Kay, S. T., et al. 2005, MNRAS, 361, 233

Bower, R. G. 1997, MNRAS, 288, 355

Collins, C. A., Guzzo, L., Böhringer, H., et al. 2000, MNRAS, 319, 939

Croston, J. H., Arnaud, M., Pointecouteau, E., \& Pratt, G. W. 2006, 459, 1007

Croton, D. J., Springel, V., White, S. D. M., et al. 2006, MNRAS, 365, 11

Dickey, J. M., \& Lockman, F. J. 1990, ARA\&A, 28, 215

Ettori, S., Tozzi, P., Borgani, S., \& Rosati, P. 2004, A\&A, 417, 13

Evrard, A. E., Metzler, C. A., \& Navarro, J. F. 1996, ApJ, 469, 494

Finoguenov, A., Borgani, S., Tornatore, L., \& Böhringer, H. 2003, A\&A, 398, L 35

Finoguenov, A., Böhringer, H., \& Zhang, Y.-Y. 2005, A\&A, 442, 827

Finoguenov, A., Davis, D. S., Zimer, M., \& Mulchaey, J. S. 2006, ApJ, 646, 143

Gray, M. E., Taylor, A. N., Meisenheimer, K., et al. 2002, ApJ, 568, 141

Haiman, Z., Allen, S., Bahcall, N., et al. 2005 [arXiv:astro-ph/0507013], white paper

Henry, J. P. 2004, ApJ, 609, 603

Ikebe, Y., Reiprich, T. H., Böhringer, H., Tanaka, Y., \& Kitayama, T. 2002, A\&A, 383,773

Kaiser, N. 1984, 1986, MNRAS, 222, 323

Kaiser, N. 1991, ApJ, 383, 104

Kay, S. T. 2004, MNRAS, 347, L13

Kay, S. T., Thomas, P. A., Jenkins, A., \& Pearce, F. R. 2004, MNRAS, 355, 1091 Kerscher, M., Mecke, K., Schuecker, P., et al. 2001, A\&A, 377, 1

Kotov, O., \& Vikhlinin, A. 2006, ApJ, 641, 752

Majumdar, S., \& Mohr, J. J. 2004, ApJ, 613, 41

Markevitch, M. 1998, ApJ, 504, 27

Muanwong, O., Thomas, P. A., Kay, S. T., \& Pearce, F. R. 2002, MNRAS, 336, 527

O’Hara, T. B., Mohr, J. J., Bialek, J. J., \& Evrard, A. E. 2006, ApJ, 639, 640

Pearce, F. R., Thomas, P. A., Couchman, H. M. P., \& Edge, A. C. 2000, MNRAS, 317,1029

Pedersen, K., \& Dahle, H. 2006 [arXiv: astro-ph/0603260]

Perlmutter, S., Aldering, G., Goldhaber, G., et al. 1999, ApJ, 517, 565

Ponman, T. J., Cannon, D. B., \& Navarro, J. F. 1999, Nature, 397, 135

Ponman, T. J., Sanderson, A. J. R., \& Finoguenov, A. 2003, MNRAS, 343, 331

Pratt, G. W., \& Arnaud, M. 2005, A\&A, 429, 791

Pratt, G. W., Arnaud, M., \& Pointecouteau, E. 2006, A\&A, 446, 429

Pratt, G. W., Böhringer, H., Croston, J. H., et al. 2007, A\&A, 461, 71

Read, A. M., \& Ponman, T. J. 2003, A\&A, 409, 395

Reiprich, T. H. 2006, A\&A, 453, L39

Reiprich, T. H., \& Böhringer, H. 2002, ApJ, 567, 716

Reiprich, T. H., Hudson, D. S., Erben, T., \& Sarazin, C. L. 2006 [arXiv:astro-ph/0603129]

Rosati, P., della Ceca, R., Norman, C., \& Giacconi, R. 1998, ApJ, 492, 21

Rosati, P., Borgani, S., \& Norman, C. 2002, ARA\&A, 40, 539

Sarazin, C. L. 1986, Rev. Mod. Phys., 58, 1

Schindler, S. 2000, A\&ASS, 142, 433

Schmidt, B. P., Suntzeff, N. B., Phillips, M. M., et al. 1998, ApJ, 507, 46

Schuecker, P., Böhringer, H., Guzzo, L., et al. 2001a, A\&A, 368, 86

Schuecker, P., Böhringer, H., Reiprich, T. H., \& Feretti, L. 2001b, A\&A, 378, 408

Schuecker, P., Guzzo, L., Collins, C. A., \& Böhringer, H. 2002, MNRAS, 335, 807 
Schuecker, P., Böhringer, H., Collins, C. A., \& Guzzo, L. 2003a, A\&A, 398, 867 Schuecker, P., Caldwell, R. R., Böhringer, H., et al. 2003b, A\&A, 402, 53 Spergel, D. N., Verde, L., Peiris, H. V., et al. 2003, ApJS, 148, 175

Spergel, D. N., Bean, R., Dore', O., et al. 2006, ApJ, submitted [arXiv: astro-ph/0603449]

Stanek, R., Evrard, A. E., Böhringer, H., Schuecker, P., \& Nord, B. 2006, ApJ, 648, 956

Tegmark, M., Eisenstein, D., Strauss, M., et al. 2006, PhRvD, 74, 123507

Trümper, J. 1993, Science, 260, 1769

Vikhlinin, A., McNamara, B. R., Forman, W., et al. 2006a, ApJ, 502, 558

Vikhlinin, A., Kravtsov, A., Forman, W., et al. 2006b, ApJ, 640, 691

Voevodkin, A., \& Vikhlinin, A. 2004, ApJ, 601, 610

Voit, M., \& Bryan, G. L. 2000, Nature, 414, 425

Voit, M., Balogh, M. L., Bower, R. G., Lacey, C. G., \& Bryan, G. L. 2003, ApJ, 593,272

Wang, \& Steinhardt 1998, ApJ, 508, 483

Zhang, Y.-Y., Böhringer, H., Finoguenov, A., et al. 2006a, A\&A, 456, 55

Zhang, Y.-Y., Finoguenov, A., Böhringer, H., et al. 2007, A\&A, 467, 437

1 Max-Planck-Institut für extraterrestrische Physik, 85748 Garching, Germany

e-mail: hxb@mpe.mpg.de

2 Service d'Astrophysique, CEA Saclay, L'Ormes des Merisiers, 91191 Gif-sur-Yvette Cedex, France

3 School of Physics and Astronomy, University of Birmingham, Edgbaston, Birmingham B15 2TT, UK

${ }^{4}$ School of Physics, Astronomy and Mathematics, University of Hertfordshire, College Lane, Hatfield AL10 9AB, UK

5 Dipartimento di Astronomia dell'Universitá di Trieste, via Tiepolo 11, 34133 Trieste, Italy

${ }^{6}$ Institute for Computational Cosmology, Department of Physics, University of Durham, South Road, Durham DH1 3LE, UK

7 Liverpool John Moores University, Liverpool, UK

8 Department of Physics and Astronomy, Michigan State University, BPS Building, East Lansing, MI 48824, USA
9 Harvard Smithsonian Center for Astrophysics, 60 Garden Street, Cambridge MA, USA

10 INAF - Osservatorio Astronomico di Brera-Merate, via Bianchi 46, 23807 Merate, Italy

11 Institute for Astronomy, University of Hawai'i, 2680 Woodlawn Drive, Honolulu, HI 96822, USA

12 Max-Planck-Institut für Radioastronomie, Auf dem Hügel 69, 53121 Bonn, Germany

13 Department of Physics, University of Illinois, 1110 West Green Street, Urbana, IL 61801, USA

14 Department of Physics, Tokyo University of Science, 1-3 Kagurazaka, Shinjyuku-ku, Tokyo 162-8601, Japan

15 Department of Astronomy, University of Michigan, 918 Dennison, 500 Church Street, Ann Arbor, MI 48109, USA

16 Department of Physics, Tokyo Metropolitan University, Hachioji, Tokyo 192-0397, Japan

17 Dark Cosmology Centre, Niels Bohr Institute, University of Copenhagen, Juliane Maries Vej 30, 2100 Copenhagen, Denmark

18 Departamento de Astronomiá y Astrofisica, Pontificia Universidad Catolica de Chile, Casilla 306, Santiago 22, Chile

19 Argelander Institute for Astronomy (AIfA), Bonn University, Auf dem Hügel 71, 53121 Bonn, Germany

20 Astronomy Centre, University of Sussex, Falmer, Brighton BN1 9QJ, UK

21 European Southern Observatory, Karl-Schwarzschild-Strasse 2, 85748 Garching, Germany

22 Department of Physics, Carnegie Mellon University, 5000 Forbes Avenue, Pittsburgh, PA 15217, USA

23 Departamento de Matemática Aplicada da Faculdade de Ciências da Universidade do Porto, Rua do Campo Alegre 687, 4169-007 Porto, Portugal

${ }^{24}$ Centro de Astrofísica da Universidade do Porto, Rua das Estrelas, 4150-762 Porto, Portugal 